\title{
SUPG-STABILIZED VIRTUAL ELEMENTS FOR DIFFUSION-CONVECTION PROBLEMS: A ROBUSTNESS ANALYSIS
}

\author{
Lourenco Beirão da Veiga ${ }^{1,2}$, Franco Dassi $^{1}$, Carlo Lovadina $^{3}$ And \\ GiUseppe VACCA ${ }^{1}$
}

\begin{abstract}
The objective of this contribution is to develop a convergence analysis for SUPG-stabilized Virtual Element Methods in diffusion-convection problems that is robust also in the convection dominated regime. For the original method introduced in [Benedetto et al., CMAME 2016] we are able to show an "almost uniform" error bound (in the sense that the unique term that depends in an unfavourable way on the parameters is damped by a higher order mesh-size multiplicative factor). We also introduce a novel discretization of the convection term that allows us to develop error estimates that are fully robust in the convection dominated cases. We finally present some numerical result.
\end{abstract}

Mathematics Subject Classification. 65N15, 65N30.

Received December 2, 2020. Accepted August 20, 2021.

\section{INTRODUCTION}

The Virtual Element Method (VEM) was introduced in [7,8] as a generalization of the Finite Element Method (FEM) to general polygonal and polyhedral meshes. Since its introduction, the VEM enjoyed a wide success in the Numerical Analysis and Engineering communities, both due to the encouraging results and the natural construction.

The possibility of using general polytopal meshes makes VEM suitable for diffusion problems, for instance, by making it much easier to adapt to complex geometries (such as in basin and reservoir simulations) and to irregularities of the solution. The VEM literature on the diffusion-reaction-convection problem is indeed very wide, covering primal and mixed methods, conforming and non-conforming schemes, ranging from foundation/theoretical contributions to more applicative articles; a very short representative list being [5,9-11,13,14,16,20,24,26,30,31]. Some examples of other numerical methods for the diffusion-reaction-convection problem that can handle polytopal meshes are $[3,4,21,22,27,28]$. On the other hand, the majority of the VEM contributions assume a dominant diffusion and do not address the significant case of convection dominated problems. Indeed, as it happens for standard FEM, unless some ad-hoc modification is introduced, also the VEM is expected to suffer in convection dominated regimes, leading to very large errors unless the mesh is extremely fine. To the best of the authors' knowledge, only in the papers $[15,17]$ such issue is addressed; in these articles a SUPG-stabilized Virtual Element

Keywords and phrases. Virtual element method, polygonal meshes, convection dominated problems, SUPG stabilization.

1 Dipartimento di Matematica e Applicazioni, Università di Milano Bicocca, Via R. Cozzi 55, 20125 Milano, Italy.

2 IMATI-CNR Pavia, Via Ferrata 1, 27100 Pavia, Italy.

3 Dipartimento di Matematica "F. Enriques", Università degli Studi di Milano, Via Cesare Saldini 50, 20133 Milano, Italy.

*Corresponding author: lourenco.beirao@unimib.it 
scheme for conforming and non-conforming VEM is proposed, and analyzed both theoretically and numerically. However, the stability and convergence analysis in $[15,17]$ is not uniform in the diffusion/convection parameters, and therefore it cannot be used to theoretically justify the method behaviour in the convection dominated regime. Moreover, a sufficiently small mesh size $h$ is required to carry out the analysis. The main difficulty in deriving uniform error estimates for SUPG-stabilized VEM is handling a variable convection coefficient in the presence of projection operators (which are needed in the VEM construction), that partially disrupt the structure of the convection term.

The aim of the present paper is to address, in the conforming case, this challenging theoretical aspect, thus deriving convergence estimates for a slight modification of the SUPG VEM scheme of [15] that are robust in the involved parameters and do not require a sufficiently small $h$ condition. We think that, in addition to filling an important theoretical gap, having this deeper understanding is fundamental in order to develop SUPG stabilizations in more complex settings, such as fluid-dynamics problems. For instance, deriving the aforementioned proofs inspired us to propose also a novel (alternative) approach for the discretization of the convective term, in addition to the original one. For the (slightly modified) discrete convection form introduced in [15], we are able to show an error estimate that is "almost uniform" in the involved parameters, in the sense that the unique term that depends in an unfavourable way on the parameters is damped by a higher order multiplicative factor in $h$. For the novel form here proposed, we are able to show full robustness in the parameters. Finally, for the sake of completeness we also present a few numerical results, the main objective being to make a practical comparison among some different discretization options described in the previous section.

The present paper is organized as follows. In Section 2 we present the continuous problem and in Section 3 we introduce some preliminaries and notation. Afterwards, in Section 4 we review the SUPG-stabilized Virtual Element Method under analysis, also introducing the novel convective term option. The main contribution of this article is Section 5, where we develop the aforementioned convergence analysis. The numerical tests are shown in Section 6.

Throughout the paper, we will follow the usual notation for Sobolev spaces and norms [1]. Hence, for an open bounded domain $\omega$, the norms in the spaces $W_{p}^{s}(\omega)$ and $L^{p}(\omega)$ are denoted by $\|\cdot\|_{W_{p}^{s}(\omega)}$ and $\|\cdot\|_{L^{p}(\omega)}$ respectively. Norm and seminorm in $H^{s}(\omega)$ are denoted respectively by $\|\cdot\|_{s, \omega}$ and $|\cdot|_{s, \omega}$, while $(\cdot, \cdot)_{\omega}$ and $\|\cdot\|_{\omega}$ denote the $L^{2}$-inner product and the $L^{2}$-norm (the subscript $\omega$ may be omitted when $\omega$ is the whole computational domain $\Omega)$.

\section{Continuous problem}

Let $\Omega \subset \mathbb{R}^{2}$ be the computational domain and let $\varepsilon>0$ represent the diffusive coefficient (assumed to be constant), while $\boldsymbol{\beta} \in\left[L^{\infty}(\Omega)\right]^{2}$ with $\operatorname{div} \boldsymbol{\beta}=0$, is the transport advective field, and $f \in L^{2}(\Omega)$ is the volume source term. Then, our linear steady advection-diffusion model problem reads

$$
\left\{\begin{array}{l}
\text { find } u \in V \text { s.t. } \\
\varepsilon a(u, v)+b(u, v)=(f, v) \quad \text { for all } v \in V,
\end{array}\right.
$$

where $V=H_{0}^{1}(\Omega)$ and the bilinear forms $a(\cdot, \cdot): V \times V \rightarrow \mathbb{R}$ and $b(\cdot, \cdot): V \times V \rightarrow \mathbb{R}$ are

$$
\begin{array}{ll}
a(u, v):=\int_{\Omega} \nabla u \cdot \nabla v \mathrm{~d} \Omega & \text { for all } u, v \in V, \\
b(u, v):=\int_{\Omega} \boldsymbol{\beta} \cdot \nabla u v \mathrm{~d} \Omega & \text { for all } u, v \in V .
\end{array}
$$

By a direct computation, being $\operatorname{div} \boldsymbol{\beta}=0$, it is easy to see that the bilinear form $b(\cdot, \cdot)$ is skew symmetric, i.e.,

$$
b(u, v)=-b(v, u) \quad \text { for all } u, v \in V .
$$


Therefore, the bilinear form $b(\cdot, \cdot)$ is equal to its skew-symmetric part, defined as:

$$
b^{\text {skew }}(u, v):=\frac{1}{2}(b(u, v)-b(v, u)) \quad \text { for all } u, v \in V .
$$

However, at the discrete level $b(\cdot, \cdot)$ and $b^{\text {skew }}(\cdot, \cdot)$ will lead to different bilinear forms, in general.

It is well known that discretizing problem (2.1) leads to instabilities when the convective term $\|\boldsymbol{\beta}\|_{\left[L^{\infty}(\Omega)\right]^{2}}$ is dominant with respect to the diffusive term $\varepsilon$ (see for instance [33]). In such situations a stabilized form of the problem is required in order to prevent spurious oscillations that can completely spoil the numerical solution. In the following sections we propose a virtual elements version of the classical Streamline Upwind Petrov Galerkin (SUPG) approach [29,32]. From now on, we assume that the material parameters are scaled so that it holds:

(A.0) Problem scaling. $\|\boldsymbol{\beta}\|_{\left[L^{\infty}(\Omega)\right]^{2}}=1$.

We finally remark that the proposed approach can be easily extended to more general situations such as reaction-convection-diffusion problems, non-constant diffusive coefficients and different boundary conditions (the main theoretical difficulties being already present in the model proposed above). Moreover, also the analysis of the three dimensional case could be developed with very similar arguments.

\section{DEFINITIONS AND PRELIMINARIES}

\subsection{SUPG stabilizing form}

From now on, we will denote with $E$ a general polygon, $e$ will denote a general edge of $E$, moreover $|E|$ and $h_{E}$ will denote the area and the diameter of $E$ respectively, whereas $\boldsymbol{n}^{E}$ will denote the unit outward normal vector to $\partial E$. Let $\left\{\Omega_{h}\right\}_{h}$ be a sequence of decompositions of $\Omega$ into general polygons $E$, where $h=\sup _{E \in \Omega_{h}} h_{E}$. We suppose that $\left\{\Omega_{h}\right\}_{h}$ fulfils the following assumption:

(A.1) Mesh assumption. There exists a positive constant $\varrho$ such that for any $E \in\left\{\Omega_{h}\right\}_{h}$

- $E$ is star-shaped with respect to a ball $B_{E}$ of radius $\geq \varrho h_{E}$;

- any edge $e$ of $E$ has length $\geq \varrho h_{E}$.

We remark that the hypotheses above, though not too restrictive in many practical cases, could possibly be further relaxed, combining the present analysis with the studies in $[6,12,19,25]$.

We now briefly review the construction of the SUPG stabilization $[29,32]$ for the advection-dominated problem (2.1). First of all, we decompose the bilinear forms $a(\cdot, \cdot)$ and $b^{\text {skew }}(\cdot, \cdot)$ into local contributions, by defining

$$
a(u, v)=: \sum_{E \in \Omega_{h}} a^{E}(u, v), \quad b^{\text {skew }}(u, v)=: \sum_{E \in \Omega_{h}} b^{\text {skew }, E}(u, v) .
$$

Let us introduce the bilinear form $\widetilde{\mathcal{A}}_{\text {supg }}^{E}(\cdot, \cdot)$, defined for all sufficiently regular functions by:

$$
\widetilde{\mathcal{A}}_{\text {supg }}^{E}(u, v):=\varepsilon a^{E}(u, v)+b^{\text {skew }, E}(u, v)+\widetilde{\mathcal{B}}^{E}(u, v)+\widetilde{\mathcal{L}}^{E}(u, v),
$$

where

$$
\begin{aligned}
& \widetilde{\mathcal{B}}^{E}(u, v):=\tau_{E} \int_{E} \boldsymbol{\beta} \cdot \nabla u(\boldsymbol{\beta} \cdot \nabla v) \mathrm{d} E \\
& \widetilde{\mathcal{L}}^{E}(u, v):=\tau_{E} \int_{E}-\varepsilon \Delta u(\boldsymbol{\beta} \cdot \nabla v) \mathrm{d} E
\end{aligned}
$$

and the SUPG parameter $\tau_{E}>0$ has to be chosen. The corresponding stabilized right-hand side $\widetilde{\mathcal{F}}_{\text {supg }}^{E}(\cdot)$ is defined by

$$
\widetilde{\mathcal{F}}_{\text {supg }}^{E}(v):=\int_{E} f v \mathrm{~d} E+\tau_{E} \int_{E} f \boldsymbol{\beta} \cdot \nabla v \mathrm{~d} E .
$$


The global approximated bilinear form $\widetilde{\mathcal{A}}_{\text {supg }}(\cdot, \cdot)$ and the global right-hand side are defined by simply summing the local contributions:

$$
\begin{aligned}
\widetilde{\mathcal{A}}_{\text {supg }}(u, v) & :=\sum_{E \in \Omega_{h}} \widetilde{\mathcal{A}}_{\text {supg }}^{E}(u, v) \\
\widetilde{\mathcal{F}}_{\text {supg }}(v) & :=\sum_{E \in \Omega_{h}} \widetilde{\mathcal{F}}_{\text {supg }}^{E}(v) .
\end{aligned}
$$

Since the exact solution $u$ of equation (2.1) satisfies $-\varepsilon \Delta u+\boldsymbol{\beta} \cdot \nabla u=f \in L^{2}(\Omega)$, then $\widetilde{\mathcal{A}}_{\text {supg }}(u, v)$ is well defined for all $v \in V$ and $u$ solves the stabilized problem

$$
\left\{\begin{array}{l}
\text { find } u \in V \text { s.t. } \\
\widetilde{\mathcal{A}}_{\text {supg }}(u, v)=\widetilde{\mathcal{F}}_{\text {supg }}(v) \quad \text { for all } v \in V .
\end{array}\right.
$$

The aim of the following sections is to derive a VEM discretization of the stabilized problem (3.7). In the following the symbol $\lesssim$ will denote a bound up to a generic positive constant, independent of the mesh size $h$, of the SUPG parameter $\tau_{E}$, of the diffusive coefficient $\varepsilon$ and of the transport advective field $\boldsymbol{\beta}$, but which may depend on $\Omega$, on the "polynomial" order of the method $k$ and on the regularity constant appearing in the mesh assumption (A.1).

\subsection{Projections and polynomial approximation properties}

In the present subsection we introduce some basic tools and notations useful in the construction and the theoretical analysis of Virtual Element Methods.

Using standard VEM notations, for $n \in \mathbb{N}, m \in \mathbb{N}$ and $p=1, \ldots, \infty$, and for any $E \in \Omega_{h}$, let us introduce the spaces:

- $\mathbb{P}_{n}(\omega)$ : the set of polynomials on $\omega$ of degree $\leq n$ (with $\left.\mathbb{P}_{-1}(\omega)=\{0\}\right)$,

- $\mathbb{P}_{n}\left(\Omega_{h}\right):=\left\{q \in L^{2}(\Omega)\right.$ s.t $\left.q\right|_{E} \in \mathbb{P}_{n}(E)$ for all $\left.E \in \Omega_{h}\right\}$,

- $W_{p}^{m}\left(\Omega_{h}\right):=\left\{v \in L^{2}(\Omega)\right.$ s.t $\left.v\right|_{E} \in W_{p}^{m}(E)$ for all $\left.E \in \Omega_{h}\right\}$ equipped with the broken norm and seminorm

$$
\begin{aligned}
\|v\|_{W_{p}^{m}\left(\Omega_{h}\right)}^{p}:=\sum_{E \in \Omega_{h}}\|v\|_{W_{p}^{m}(E)}^{p}, & |v|_{W_{p}^{m}\left(\Omega_{h}\right)}^{p}:=\sum_{E \in \Omega_{h}}|v|_{W_{p}^{m}(E)}^{p}, \quad \text { if } 1 \leq p<\infty, \\
\|v\|_{W_{p}^{m}\left(\Omega_{h}\right)}:=\max _{E \in \Omega_{h}}\|v\|_{W_{p}^{m}(E)}, & |v|_{W_{p}^{m}\left(\Omega_{h}\right)}:=\max _{E \in \Omega_{h}}|v|_{W_{p}^{m}(E)}, \quad \text { if } p=\infty,
\end{aligned}
$$

and the following polynomial projections:

- the $\boldsymbol{L}^{\mathbf{2}}$-projection $\Pi_{n}^{0, E}: L^{2}(E) \rightarrow \mathbb{P}_{n}(E)$, given by

$$
\int_{E} q_{n}\left(v-\Pi_{n}^{0, E} v\right) \mathrm{d} E=0 \quad \text { for all } v \in L^{2}(E) \text { and } q_{n} \in \mathbb{P}_{n}(E),
$$

with obvious extension for vector functions $\Pi_{n}^{0, E}:\left[L^{2}(E)\right]^{2} \rightarrow\left[\mathbb{P}_{n}(E)\right]^{2}$;

- the $\boldsymbol{H}^{\mathbf{1}}$-seminorm projection $\Pi_{n}^{\nabla, E}: H^{1}(E) \rightarrow \mathbb{P}_{n}(E)$, defined by

$$
\left\{\begin{array}{l}
\int_{E} \nabla q_{n} \cdot \nabla\left(v-\Pi_{n}^{\nabla, E} v\right) \mathrm{d} E=0 \quad \text { for all } v \in H^{1}(E) \text { and } q_{n} \in \mathbb{P}_{n}(E), \\
\int_{\partial E}\left(v-\Pi_{n}^{\nabla, E} v\right) \mathrm{d} s=0,
\end{array}\right.
$$

with global counterparts $\Pi_{n}^{0}: L^{2}(\Omega) \rightarrow \mathbb{P}_{n}\left(\Omega_{h}\right)$ and $\Pi_{n}^{\nabla}: H^{1}\left(\Omega_{h}\right) \rightarrow \mathbb{P}_{n}\left(\Omega_{h}\right)$ defined by

$$
\left.\left(\Pi_{n}^{0} v\right)\right|_{E}=\Pi_{n}^{0, E} v,\left.\quad\left(\Pi_{n}^{\nabla} v\right)\right|_{E}=\Pi_{n}^{\nabla, E} v, \quad \text { for all } E \in \Omega_{h} .
$$

We finally mention two classical results for polynomials on star-shaped domains (see for instance [18]). 
Lemma 3.1 (Bramble-Hilbert). Under the assumption (A.1), for any $E \in \Omega_{h}$ and for any smooth enough function $\varphi$ defined on $E$, it holds

$$
\begin{array}{lr}
\left\|\varphi-\Pi_{n}^{0, E} \varphi\right\|_{W_{p}^{m}(E)} \lesssim h_{E}^{s-m}|\varphi|_{W_{p}^{s}(E)} \quad s, m \in \mathbb{N}, m \leq s \leq n+1, p \in[1+\infty] \\
\left\|\varphi-\Pi_{n}^{\nabla, E} \varphi\right\|_{m, E} \lesssim h_{E}^{s-m}|\varphi|_{s, E} \quad s, m \in \mathbb{N}, m \leq s \leq n+1, s \geq 1 .
\end{array}
$$

Lemma 3.2 (Inverse estimate). Let, for any $E \in \Omega_{h}, \gamma_{E}$ denote the smallest positive constant such that for any $\boldsymbol{p}_{n} \in\left[\mathbb{P}_{n}(E)\right]^{2}$, it holds

$$
\left\|\operatorname{div} \boldsymbol{p}_{n}\right\|_{0, E}^{2} \leq \gamma_{E} h_{E}^{-2}\left\|\boldsymbol{p}_{n}\right\|_{0, E}^{2}
$$

Then, under assumption (A.1), there exists $\gamma \in \mathbb{R}^{+}$such that $\gamma_{E} \leq \gamma$ for all $E \in\left\{\Omega_{h}\right\}_{h}$.

\section{Virtual ElEMENT DiscRetization}

\subsection{Virtual element spaces}

Let $k \geq 1$ be the "polynomial" order of the method. For any $E \in \Omega_{h}$ we consider the local "enhanced" virtual element space [2] given by

$$
\begin{aligned}
& V_{h}(E)=\left\{v_{h} \in H^{1}(E) \cap C^{0}(\partial E) \quad\right. \text { s.t. }\left.v_{h}\right|_{e} \in \mathbb{P}_{k}(e) \quad \text { for all } e \in \partial E, \\
&\left.\Delta v_{h} \in \mathbb{P}_{k}(E), \quad\left(v_{h}-\Pi_{k}^{\nabla, E} v_{h}, \widehat{p}_{k}\right)=0 \quad \text { for all } \widehat{p}_{k} \in \mathbb{P}_{k}(E) / \mathbb{P}_{k-2}(E)\right\} .
\end{aligned}
$$

We here summarize the main properties of the space $V_{h}(E)$ (we refer to [2] for a deeper analysis).

(P.1) Polynomial inclusion: $\mathbb{P}_{k}(E) \subseteq V_{h}(E)$;

(P.2) Degrees of freedom: the following linear operators $\mathbf{D}_{\mathbf{V}}$ constitute a set of DoFs for $V_{h}(E)$ :

$\mathbf{D}_{\mathbf{V}} \mathbf{1}$ the values of $v_{h}$ at the vertexes of the polygon $E$,

$\mathbf{D}_{\mathbf{V}} \mathbf{2}$ the values of $v_{h}$ at $k-1$ distinct points of every edge $e \in \partial E$,

$\mathbf{D}_{\mathbf{V}} \mathbf{3}$ the moments up to order $k-2$ of $v_{h}$ in $E$ :

$$
\frac{1}{|E|} \int_{E} v_{h} m_{i} \mathrm{~d} E
$$

where $\left\{m_{i}\right\}_{i}$ is a polynomial basis of $\mathbb{P}_{k-2}(E)$ s.t. $\left\|m_{i}\right\|_{L^{\infty}(E)}=1$;

(P.3) Polynomial projections: the DoFs $\mathbf{D}_{\mathrm{V}}$ allow us to compute the following linear operators:

$$
\Pi_{k}^{\nabla, E}: V_{h}(E) \rightarrow \mathbb{P}_{k}(E), \quad \Pi_{k}^{0, E}: V_{h}(E) \rightarrow \mathbb{P}_{k}(E), \quad \Pi_{k}^{0, E}: \nabla V_{h}(E) \rightarrow\left[\mathbb{P}_{k}(E)\right]^{2} .
$$

The global virtual element space is obtained by gluing such local spaces, i.e.,

$$
V_{h}\left(\Omega_{h}\right)=\left\{v_{h} \in V \quad \text { s.t. }\left.\quad v_{h}\right|_{E} \in V_{h}(E) \text { for all } E \in \Omega_{h}\right\}
$$

with the associated set of degrees of freedom.

We finally recall from $[19,23]$ the optimal approximation property for the space $V_{h}\left(\Omega_{h}\right)$.

Lemma 4.1 (Approximation using virtual element functions). Under the assumption (A.1) for any $v \in V \cap$ $H^{s+1}\left(\Omega_{h}\right)$ there exists $v_{\mathcal{I}} \in V_{h}\left(\Omega_{h}\right)$ such that for all $E \in \Omega_{h}$ it holds

$$
\left\|v-v_{\mathcal{I}}\right\|_{0, E}+h_{E}\left\|\nabla\left(v-v_{\mathcal{I}}\right)\right\|_{0, E} \lesssim h_{E}^{s+1}|v|_{s+1, E}
$$

where $0<s \leq k$. 


\subsection{Virtual element forms}

The next step in the construction of our method is to define a discrete versions of the stabilized SUPG form $\widetilde{\mathcal{A}}_{\text {supg }}(\cdot, \cdot)$ in (3.1). It is clear that for an arbitrary pair $\left(u_{h}, v_{h}\right) \in V_{h}(E) \times V_{h}(E)$, the quantity $\widetilde{\mathcal{A}}_{\text {supg }}^{E}\left(u_{h}, v_{h}\right)$ is not computable since $u_{h}$ and $v_{h}$ are not known in closed form. Therefore, following the usual procedure in the VEM setting, we need to construct a computable discrete bilinear form. $\widetilde{\mathcal{A}}_{\text {supg }}^{E}$.

In the following, in accordance with definition (3.1), we define a discrete counterpart of each term composing

Exploiting property (P.3), let $a_{h}^{E}(\cdot, \cdot): V_{h}(E) \times V_{h}(E) \rightarrow \mathbb{R}$ be a computable approximation of the continuous form $a^{E}(\cdot, \cdot)$, defined for all $u_{h}, v_{h} \in V_{h}(E)$ by

$$
a_{h}^{E}\left(u_{h}, v_{h}\right):=\int_{E} \boldsymbol{\Pi}_{k-1}^{0, E} \nabla u_{h} \cdot \boldsymbol{\Pi}_{k-1}^{0, E} \nabla v_{h} \mathrm{~d} E+\mathcal{S}^{E}\left(\left(I-\Pi_{k}^{\nabla, E}\right) u_{h},\left(I-\Pi_{k}^{\nabla, E}\right) v_{h}\right) .
$$

Here, the stabilizing bilinear form $\mathcal{S}^{E}(\cdot, \cdot): V_{h}(E) \times V_{h}(E) \rightarrow \mathbb{R}$ satisfies

$$
\alpha_{*}\left|v_{h}\right|_{1, E}^{2} \leq \mathcal{S}^{E}\left(v_{h}, v_{h}\right) \leq \alpha^{*}\left|v_{h}\right|_{1, E}^{2} \quad \text { for all } v_{h} \in \operatorname{Ker}\left(\Pi_{k}^{\nabla, E}\right)
$$

for two positive uniform constants $\alpha_{*}$ and $\alpha^{*}$. The condition above essentially requires the stabilizing term $\mathcal{S}^{E}\left(v_{h}, v_{h}\right)$ to scale as $\left|v_{h}\right|_{1, E}^{2}$. For instance, the standard choices for the stabilization are the dofi-dofi stabilization [7] and the D-recipe stabilization introduced in [11].

Concerning the approximation of the convective term $b^{E}(\cdot, \cdot)$, we here propose two possible choices: recalling property (P.3), let us define for all $u_{h}, v_{h} \in V_{h}(E)$ the following computable bilinear forms

$$
\begin{aligned}
b_{o, h}^{E}\left(u_{h}, v_{h}\right) & :=\int_{E} \boldsymbol{\beta} \cdot \boldsymbol{\Pi}_{k}^{0, E} \nabla u_{h} \Pi_{k}^{0, E} v_{h} \mathrm{~d} E \\
b_{\partial, h}^{E}\left(u_{h}, v_{h}\right) & :=\int_{E} \boldsymbol{\beta} \cdot \nabla \Pi_{k}^{0, E} u_{h} \Pi_{k}^{0, E} v_{h} \mathrm{~d} E+\int_{\partial E}\left(\boldsymbol{\beta} \cdot \boldsymbol{n}^{E}\right)\left(I-\Pi_{k}^{0, E}\right) u_{h} \Pi_{k}^{0, E} v_{h} \mathrm{~d} s .
\end{aligned}
$$

The form (4.5) follows a more standard "approximation by projection" VEM approach (see for instance [15]) and is based on a higher order projection of the gradient. The novel form (4.6) encompasses a boundary integral term and is amenable to the development of an improved theoretical result ( $c f$. Propositions 5.3 and 5.4). In the following $b_{h}^{E}(\cdot, \cdot): V_{h}(E) \times V_{h}(E) \rightarrow \mathbb{R}$ will denote indifferently one of the aforementioned forms and, in accordance with (2.4), for all $u_{h}, v_{h} \in V_{h}(E)$ we define

$$
b_{h}^{\text {skew }, E}\left(u_{h}, v_{h}\right):=\frac{1}{2}\left(b_{h}^{E}\left(u_{h}, v_{h}\right)-b_{h}^{E}\left(v_{h}, u_{h}\right)\right) .
$$

Exploiting again property (P.3), the stabilized forms $\widetilde{\mathcal{B}}^{E}(\cdot, \cdot)$ in $(3.2)$ and $\widetilde{\mathcal{L}}^{E}(\cdot, \cdot)$ in $(3.3)$ are discretized as follows

$$
\begin{aligned}
& \mathcal{B}^{E}\left(u_{h}, v_{h}\right):=\tau_{E} \int_{E} \boldsymbol{\beta} \cdot \boldsymbol{\Pi}_{k-1}^{0, E} \nabla u_{h} \boldsymbol{\beta} \cdot \boldsymbol{\Pi}_{k-1}^{0, E} \nabla v_{h} \mathrm{~d} E+\tau_{E} \beta_{E}^{2} \mathcal{S}^{E}\left(\left(I-\Pi_{k}^{\nabla, E}\right) u_{h},\left(I-\Pi_{k}^{\nabla, E}\right) v_{h}\right) \\
& \mathcal{L}^{E}\left(u_{h}, v_{h}\right):=\tau_{E} \int_{E}-\varepsilon \operatorname{div} \boldsymbol{\Pi}_{k-1}^{0, E} \nabla u_{h} \boldsymbol{\beta} \cdot \boldsymbol{\Pi}_{k-1}^{0, E} \nabla v_{h} \mathrm{~d} E
\end{aligned}
$$

where $\beta_{E}:=\|\boldsymbol{\beta}\|_{\left[L^{\infty}(E)\right]^{2}}$ and the parameter $\tau_{E}>0$ has to be chosen.

In accordance with (3.1), the VEM stabilized form $\mathcal{A}_{\text {supg }}^{E}(\cdot, \cdot): V_{h}(E) \times V_{h}(E) \rightarrow \mathbb{R}$ is defined by

$$
\mathcal{A}_{\text {supg }}^{E}\left(u_{h}, v_{h}\right):=\varepsilon a_{h}^{E}\left(u_{h}, v_{h}\right)+b_{h}^{\text {skew }, E}\left(u_{h}, v_{h}\right)+\mathcal{B}^{E}\left(u_{h}, v_{h}\right)+\mathcal{L}^{E}\left(u_{h}, v_{h}\right)
$$

for all $u_{h}, v_{h} \in V_{h}(E)$. 
The global approximated bilinear form $\mathcal{A}_{\text {supg }}(\cdot, \cdot): V_{h}\left(\Omega_{h}\right) \times V_{h}\left(\Omega_{h}\right) \rightarrow \mathbb{R}$ is thus defined by summing the local contributions, i.e.,

$$
\mathcal{A}_{\text {supg }}(u, v):=\sum_{E \in \Omega_{h}} \mathcal{A}_{\text {supg }}^{E}\left(u_{h}, v_{h}\right) \quad \text { for all } u_{h}, v_{h} \in V_{h}\left(\Omega_{h}\right) .
$$

The corresponding computable VEM version of the SUPG right-hand side in (3.4) reads as

$$
\mathcal{F}_{\text {supg }}^{E}\left(v_{h}\right):=\int_{E} f \Pi_{k}^{0, E} v_{h} \mathrm{~d} E+\tau_{E} \int_{E} f \boldsymbol{\beta} \cdot \boldsymbol{\Pi}_{k-1}^{0, E} \nabla v_{h} \mathrm{~d} E
$$

and its global counterpart is

$$
\mathcal{F}_{\text {supg }}\left(v_{h}\right):=\sum_{E \in \Omega_{h}} \mathcal{F}_{\text {supg }}^{E}\left(v_{h}\right) \quad \text { for all } v_{h} \in V_{h}\left(\Omega_{h}\right) .
$$

\subsection{Virtual element SUPG problem}

Referring to the discrete space (4.2), the discrete bilinear form (4.11) and the approximated right-hand side (4.13), the virtual element SUPG approximation of the advection-dominated diffusion equation (2.1) is

$$
\left\{\begin{array}{l}
\text { find } u_{h} \in V_{h}\left(\Omega_{h}\right) \text { s.t. } \\
\mathcal{A}_{\text {supg }}\left(u_{h}, v_{h}\right)=\mathcal{F}_{\text {supg }}\left(v_{h}\right) \quad \text { for all } v_{h} \in V_{h}\left(\Omega_{h}\right) .
\end{array}\right.
$$

\section{TheOreticAl ANALYSis}

In this section we analyze the stabilization method defined in (4.14). In particular, we assess the stability property of problem (4.14) and we provide the convergence error estimate for the discrete solution obtained with both discrete convective forms defined in (4.5) and (4.6). All estimates clearly display the dependence on the mesh size $h$, on the parameter $\tau_{E}$ and the problem data $\varepsilon$ and $\boldsymbol{\beta}$.

\subsection{Stability}

Let us start with the stability analysis for the proposed VEM SUPG method. First of all we define the VEM SUPG norm

$$
\left\|v_{h}\right\|_{\mathrm{supg}, E}^{2}:=\varepsilon\left\|\nabla v_{h}\right\|_{0, E}^{2}+\tau_{E}\left\|\boldsymbol{\beta} \cdot \boldsymbol{\Pi}_{k-1}^{0, E} \nabla v_{h}\right\|_{0, E}^{2}+\tau_{E} \beta_{E}^{2}\left\|\nabla\left(I-\Pi_{k}^{\nabla, E}\right) v_{h}\right\|_{0, E}^{2}
$$

with global counterpart

$$
\left\|v_{h}\right\|_{\text {supg }}^{2}:=\sum_{E \in \Omega_{h}}\left\|v_{h}\right\|_{\text {supg }, E}^{2}
$$

Proposition 5.1 (Coercivity). Under the assumption (A.1) if the parameters $\tau_{E}$ satisfy

$$
\tau_{E} \leq \frac{h_{E}^{2}}{\varepsilon \gamma_{E}} \quad \forall E \in \Omega_{h}
$$

where $\gamma_{E}$ is the constant appearing in the inverse estimate of Lemma 3.2, the bilinear form $\mathcal{A}_{\mathrm{supg}}^{E}(\cdot, \cdot)$ satisfies for all $v_{h} \in V_{h}(E)$ the coerciveness inequality

$$
\left\|v_{h}\right\|_{\text {supg }, E}^{2} \lesssim \mathcal{A}_{\text {supg }}^{E}\left(v_{h}, v_{h}\right) .
$$


Proof. We simply consider all the terms in the sum (4.10). For the first three terms by definitions (4.3), (4.7) and (4.8) and stability estimate (4.4) we get

$$
\begin{aligned}
\varepsilon a_{h}^{E}\left(v_{h}, v_{h}\right) & \geq \varepsilon\left\|\boldsymbol{\Pi}_{k-1}^{0, E} \nabla v_{h}\right\|_{0, E}^{2}+\varepsilon \alpha_{*}\left\|\nabla v_{h}-\nabla \Pi_{k}^{\nabla, E} v_{h}\right\|_{0, E}^{2} \\
b_{h}^{\text {skew }, E}\left(v_{h}, v_{h}\right) & =0 \\
\mathcal{B}^{E}\left(v_{h}, v_{h}\right) & \geq \tau_{E}\left\|\boldsymbol{\beta} \cdot \boldsymbol{\Pi}_{k-1}^{0, E} \nabla v_{h}\right\|_{0, E}^{2}+\tau_{E} \beta_{E}^{2} \alpha_{*}\left\|\nabla v_{h}-\nabla \Pi_{k}^{\nabla, E} v_{h}\right\|_{0, E}^{2}
\end{aligned}
$$

whereas for the last term we infer

$$
\begin{array}{rlr}
\mathcal{L}^{E}\left(v_{h}, v_{h}\right) & =\tau_{E} \int_{E}-\varepsilon \operatorname{div} \boldsymbol{\Pi}_{k-1}^{0, E} \nabla v_{h} \boldsymbol{\beta} \cdot \boldsymbol{\Pi}_{k-1}^{0, E} \nabla v_{h} \mathrm{~d} E & \\
& \geq-\tau_{E}\left\|\varepsilon \operatorname{div} \boldsymbol{\Pi}_{k-1}^{0, E} \nabla v_{h}\right\|_{0, E}\left\|\boldsymbol{\beta} \cdot \boldsymbol{\Pi}_{k-1}^{0, E} \nabla v_{h}\right\|_{0, E}^{2} & \text { (Cauchy-Schwarz) } \\
& \geq-\frac{1}{2} \tau_{E} \varepsilon^{2}\left\|\operatorname{div} \boldsymbol{\Pi}_{k-1}^{0, E} \nabla v_{h}\right\|_{0, E}^{2}-\frac{1}{2} \tau_{E}\left\|\boldsymbol{\beta} \cdot \boldsymbol{\Pi}_{k-1}^{0, E} \nabla v_{h}\right\|_{0, E}^{2} & \text { (arith.-geom. mean) } \\
& \geq-\frac{1}{2} \tau_{E} \gamma_{E} h_{E}^{-2} \varepsilon^{2}\left\|\boldsymbol{\Pi}_{k-1}^{0, E} \nabla v_{h}\right\|_{0, E}^{2}-\frac{1}{2} \tau_{E}\left\|\boldsymbol{\beta} \cdot \boldsymbol{\Pi}_{k-1}^{0, E} \nabla v_{h}\right\|_{0, E}^{2} & \text { (Lemma 3.2) } \\
& \geq-\frac{1}{2} \varepsilon\left\|\boldsymbol{\Pi}_{k-1}^{0, E} \nabla v_{h}\right\|_{0, E}^{2}-\frac{1}{2} \tau_{E}\left\|\boldsymbol{\beta} \cdot \boldsymbol{\Pi}_{k-1}^{0, E} \nabla v_{h}\right\|_{0, E}^{2} .
\end{array}
$$

Moreover, by definition of $L^{2}$-orthogonal projection (3.8), being $\nabla \Pi_{k}^{\nabla, E} v_{h} \in\left[\mathbb{P}_{k-1}(E)\right]^{2}$, it holds

$$
\left\|\nabla v_{h}-\nabla \Pi_{k}^{\nabla, E} v_{h}\right\|_{0, E}^{2} \geq\left\|\nabla v_{h}-\Pi_{k-1}^{0, E} \nabla v_{h}\right\|_{0, E}^{2} .
$$

Collecting the previous bound, (5.4) and (5.5) we obtain

$$
\begin{aligned}
\mathcal{A}_{\text {supg }}^{E}\left(v_{h}, v_{h}\right) \geq & \frac{1}{2} \varepsilon\left\|\boldsymbol{\Pi}_{k-1}^{0, E} \nabla v_{h}\right\|_{0, E}^{2}+\frac{1}{2} \tau_{E}\left\|\boldsymbol{\beta} \cdot \boldsymbol{\Pi}_{k-1}^{0, E} \nabla v_{h}\right\|_{0, E}^{2}+ \\
& +\alpha_{*} \varepsilon\left\|\nabla v_{h}-\boldsymbol{\Pi}_{k-1}^{0, E} \nabla v_{h}\right\|_{0, E}^{2}+\alpha_{*} \tau_{E} \beta_{E}^{2}\left\|\nabla\left(I-\Pi_{k}^{\nabla, E}\right) v_{h}\right\|_{0, E}^{2} \\
\geq & \min \left\{\frac{1}{2}, \alpha_{*}\right\}\left\|v_{h}\right\|_{\text {supg }, E}^{2} .
\end{aligned}
$$

Remark 5.1. Notice that the norm $\|\cdot\|_{\text {supg, } E}$ is slightly different from the usual norm introduced in standard SUPG theory $[29,32]$, i.e.,

$$
\left\|v_{h}\right\|_{\widetilde{\text { supg }, E}}^{2}:=\varepsilon\left\|\nabla v_{h}\right\|_{0, E}^{2}+\tau_{E}\left\|\boldsymbol{\beta} \cdot \nabla v_{h}\right\|_{0, E}^{2} .
$$

However we observe that the "classical norm" $\|\cdot\|_{\widetilde{\text { supg }, E}}^{2}$ is controlled by the "VEM norm" $\|\cdot\|_{\text {supg, } E}$. Indeed, recalling (5.6), for any $v_{h} \in H^{1}(E)$ it holds

$$
\begin{aligned}
\left\|\boldsymbol{\beta} \cdot \nabla v_{h}\right\|_{0, E}^{2} & \leq 2\left\|\boldsymbol{\beta} \cdot \boldsymbol{\Pi}_{k-1}^{0, E} \nabla v_{h}\right\|_{0, E}^{2}+2 \beta_{E}^{2}\left\|\left(I-\boldsymbol{\Pi}_{k-1}^{0, E}\right) \nabla v_{h}\right\|_{0, E}^{2} \\
& \leq 2\left\|\boldsymbol{\beta} \cdot \boldsymbol{\Pi}_{k-1}^{0, E} \nabla v_{h}\right\|_{0, E}^{2}+2 \beta_{E}^{2}\left\|\nabla\left(I-\Pi_{k}^{0, E}\right) v_{h}\right\|_{0, E}^{2} .
\end{aligned}
$$

\subsection{Error estimates}

The aim of the present section is to derive the rate of convergence for the proposed SUPG virtual element scheme (4.14) in terms of the mesh size $h$, the SUPG parameter $\tau_{E}$, the diffusive coefficient $\varepsilon$ and transport advective field $\boldsymbol{\beta}$. The hidden constants may depend on $\Omega$, on $k$, on the regularity constant appearing in the mesh assumption (A.1) and on the stability constants $\alpha_{*}$ and $\alpha^{*}$ (cf. (4.4)).

Let $u \in V$ and $u_{h} \in V_{h}\left(\Omega_{h}\right)$ be the solutions of problems (3.7) and (4.14), respectively, and let us define the following error functions

$$
e_{\mathcal{I}}:=u-u_{\mathcal{I}}, \quad e_{\pi}:=u-\Pi_{k}^{\nabla} u, \quad e_{h}:=u_{h}-u_{\mathcal{I}},
$$


where $u_{\mathcal{I}} \in V_{h}\left(\Omega_{h}\right)$ is the interpolant function of $u$ defined in Lemma 4.1, and $\Pi_{k}^{\nabla} u \in \mathbb{P}_{k}\left(\Omega_{h}\right)$ is the piecewise polynomial defined in (3.10). We introduce the analysis with the following abstract error estimation.

Proposition 5.2. Let $u \in V$ and $u_{h} \in V_{h}\left(\Omega_{h}\right)$ be the solutions of problems (3.7) and (4.14), respectively. Then under assumption (A.1) if the parameters $\tau_{E}$ satisfy (5.3), it holds that

$$
\left\|u-u_{h}\right\|_{\text {supg }}^{2} \lesssim\left\|e_{\mathcal{I}}\right\|_{\text {supg }}^{2}+\sum_{E \in \Omega_{h}}\left(\eta_{\mathcal{F}}^{E}+\eta_{a}^{E}+\eta_{b}^{E}+\eta_{\mathcal{B}}^{E}+\eta_{\mathcal{L}}^{E}\right)
$$

where

$$
\begin{aligned}
\eta_{\mathcal{F}}^{E} & :=\widetilde{\mathcal{F}}_{\text {supg }}^{E}\left(e_{h}\right)-\mathcal{F}_{\text {supg }}^{E}\left(e_{h}\right) \\
\eta_{a}^{E} & :=\varepsilon a^{E}\left(u, e_{h}\right)-\varepsilon a_{h}^{E}\left(u_{\mathcal{I}}, e_{h}\right) \\
\eta_{b}^{E} & :=b^{\text {skew }, E}\left(u, e_{h}\right)-b_{h}^{\text {skew }, E}\left(u_{\mathcal{I}}, e_{h}\right) \\
\eta_{\mathcal{B}}^{E} & :=\widetilde{\mathcal{B}}^{E}\left(u, e_{h}\right)-\mathcal{B}^{E}\left(u_{\mathcal{I}}, e_{h}\right) \\
\eta_{\mathcal{L}}^{E} & :=\widetilde{\mathcal{L}}^{E}\left(u, e_{h}\right)-\mathcal{L}^{E}\left(u_{\mathcal{I}}, e_{h}\right)
\end{aligned}
$$

Proof. Simple computations yield

$$
\begin{aligned}
\left\|e_{h}\right\|_{\text {supg }}^{2} & \lesssim \mathcal{A}_{\text {supg }}\left(e_{h}, e_{h}\right)=\mathcal{A}_{\text {supg }}\left(u_{h}-u_{\mathcal{I}}, e_{h}\right) \\
& \lesssim \mathcal{F}_{\text {supg }}\left(e_{h}\right)-\widetilde{\mathcal{F}}_{\text {supg }}\left(e_{h}\right)+\widetilde{\mathcal{A}}_{\text {supg }}\left(u, e_{h}\right)-\mathcal{A}_{\text {supg }}\left(u_{\mathcal{I}}, e_{h}\right) \\
& \lesssim \sum_{E \in \Omega_{h}}\left(\eta_{\mathcal{F}}^{E}+\eta_{a}^{E}+\eta_{b}^{E}+\eta_{\mathcal{B}}^{E}+\eta_{\mathcal{L}}^{E}\right)
\end{aligned}
$$

The thesis now follows by the triangular inequality.

The next step in the analysis consists in estimating all the terms in the bound (5.7). We make the following assumption:

(A.2) Data assumption. The solution $u$, the advective field $\boldsymbol{\beta}$ and the load $f$ in (3.7) satisfy:

$$
u \in H^{s+1}\left(\Omega_{h}\right), \quad f \in H^{s+1}\left(\Omega_{h}\right), \quad \boldsymbol{\beta} \in\left[W_{\infty}^{s+1}\left(\Omega_{h}\right)\right]^{2},
$$

for some $0<s \leq k$.

Note that in the following lemmas it is not restrictive to assume $\beta_{E}>0$ since $\beta_{E}=0$ implies $\left.\boldsymbol{\beta}\right|_{E}=0$ and thus the corresponding terms vanish.

Lemma 5.1 (Estimate of $\left.\left\|e_{\mathcal{I}}\right\|_{\text {supg }}\right)$. Under assumptions (A.1) and (A.2), the term $\left\|e_{\mathcal{I}}\right\|_{\text {supg }}^{2}$ can be bounded as follows (for $0<s \leq k$ )

$$
\left\|e_{\mathcal{I}}\right\|_{\text {supg }}^{2} \lesssim \sum_{E \in \Omega_{h}}\left(\varepsilon+\tau_{E} \beta_{E}^{2}\right) h_{E}^{2 s}|u|_{s+1, E}^{2}
$$

Proof. Applying the definition of the norm $\|\cdot\|_{\text {supg, } E}$, of the $L^{2}$-orthogonal projection $\boldsymbol{\Pi}_{k-1}^{0, E}(c f$. (3.8)), of the $H^{1}$-orthogonal projection $\Pi_{k}^{\nabla, E}(c f .(3.9))$, and the interpolation estimate of Lemma 4.1, we easily obtain

$$
\begin{aligned}
\left\|e_{\mathcal{I}}\right\|_{\text {supg }, E}^{2} & =\varepsilon\left\|\nabla e_{\mathcal{I}}\right\|_{0, E}^{2}+\tau_{E}\left\|\boldsymbol{\beta} \cdot \boldsymbol{\Pi}_{k-1}^{0, E} \nabla e_{\mathcal{I}}\right\|_{0, E}^{2}+\tau_{E} \beta_{E}^{2}\left\|\nabla\left(I-\Pi_{k}^{\nabla, E}\right) e_{\mathcal{I}}\right\|_{0, E}^{2} \\
& \leq \varepsilon\left\|\nabla e_{\mathcal{I}}\right\|_{0, E}^{2}+\tau_{E} \beta_{E}^{2}\left\|\nabla e_{\mathcal{I}}\right\|_{0, E}^{2}+\tau_{E} \beta_{E}^{2}\left\|\nabla e_{\mathcal{I}}\right\|_{0, E}^{2} \lesssim\left(\varepsilon+\tau_{E} \beta_{E}^{2}\right)\left\|\nabla e_{\mathcal{I}}\right\|_{0, E}^{2} \\
& \lesssim\left(\varepsilon+\tau_{E} \beta_{E}^{2}\right) h_{E}^{2 s}|u|_{s+1, E}^{2} .
\end{aligned}
$$

The thesis now follows by summing the local contributions. 
Lemma 5.2 (Estimate of $\left.\eta_{\mathcal{F}}^{E}\left(e_{h}\right)\right)$. Under the assumptions (A.1) and (A.2), the term $\eta_{\mathcal{F}}^{E}$ can be bounded as follows (for $0<s \leq k$ )

$$
\eta_{\mathcal{F}}^{E} \lesssim\left(\lambda_{E} h_{E}^{s+2}|f|_{s+1, E}+\tau_{E}^{1 / 2} \frac{\|\boldsymbol{\beta}\|_{\left[W_{\infty}^{s}(E)\right]^{2}}}{\beta_{E}} h_{E}^{s}\|f\|_{s, E}\right)\left\|e_{h}\right\|_{\text {supg }, E}
$$

where for any $E \in \Omega_{h}$

$$
\lambda_{E}:=\min \left\{\frac{1}{\beta_{E} \tau_{E}^{1 / 2}}, \frac{1}{\varepsilon^{1 / 2}}\right\} .
$$

Proof. Applying the definitions (3.4), (4.12) and the definition of $L^{2}$-orthogonal projection we obtain

$$
\begin{aligned}
\eta_{\mathcal{F}}^{E} & =\widetilde{\mathcal{F}}_{\text {supg }}^{E}\left(e_{h}\right)-\mathcal{F}_{\text {supg }}^{E}\left(e_{h}\right) \\
& =\left(f, e_{h}-\Pi_{k}^{0, E} e_{h}\right)_{0, E}+\tau_{E}\left(f, \boldsymbol{\beta} \cdot\left(\nabla e_{h}-\boldsymbol{\Pi}_{k-1}^{0, E} \nabla e_{h}\right)\right)_{0, E} \\
& =\left(\left(I-\Pi_{k}^{0, E}\right) f,\left(I-\Pi_{k}^{0, E}\right) e_{h}\right)_{0, E}+\tau_{E}\left(f \boldsymbol{\beta},\left(I-\boldsymbol{\Pi}_{k-1}^{0, E}\right) \nabla e_{h}\right)_{0, E} \\
& =\left(\left(I-\Pi_{k}^{0, E}\right) f,\left(I-\Pi_{k}^{\nabla, E}\right) e_{h}\right)_{0, E}+\tau_{E}\left(\left(I-\boldsymbol{\Pi}_{k-1}^{0, E}\right) f \boldsymbol{\beta},\left(I-\boldsymbol{\Pi}_{k-1}^{0, E}\right) \nabla e_{h}\right)_{0, E} \\
& =: \eta_{\mathcal{F}, 1}^{E}+\eta_{\mathcal{F}, 2}^{E} .
\end{aligned}
$$

Using a scaled Poincaré inequality we infer

$$
\eta_{\mathcal{F}, 1}^{E} \leq\left\|\left(I-\Pi_{k}^{0, E}\right) f\right\|_{0, E}\left\|\left(I-\Pi_{k}^{\nabla, E}\right) e_{h}\right\|_{0, E} \lesssim h_{E}\left\|\left(I-\Pi_{k}^{0, E}\right) f\right\|_{0, E}\left\|\nabla\left(I-\Pi_{k}^{\nabla, E}\right) e_{h}\right\|_{0, E} .
$$

Recalling the definition of the norm $\|\cdot\|_{\text {supg, } E}$ and the stability of $\Pi_{k}^{\nabla, E}$ with respect to the $H^{1}$-seminorm, from Lemma 3.1 we get

$$
\eta_{\mathcal{F}, 1}^{E} \lesssim \min \left\{\frac{1}{\beta_{E} \tau_{E}^{1 / 2}}, \frac{1}{\varepsilon^{1 / 2}}\right\} h_{E}^{s+2}|f|_{s+1, E}\left\|e_{h}\right\|_{\text {supg }, E} .
$$

Regarding the second term $\eta_{\mathcal{F}, 2}^{E}$, from (5.6) and Lemma 3.1 we obtain

$$
\begin{aligned}
\eta_{\mathcal{F}, 2}^{E} & \leq \tau_{E}\left\|\left(I-\boldsymbol{\Pi}_{k-1}^{0, E}\right) f \boldsymbol{\beta}\right\|_{0, E}\left\|\left(I-\boldsymbol{\Pi}_{k-1}^{0, E}\right) \nabla e_{h}\right\|_{0, E} \lesssim \tau_{E}^{1 / 2} \frac{\left\|\left(I-\boldsymbol{\Pi}_{k-1}^{0, E}\right) f \boldsymbol{\beta}\right\|_{0, E}}{\beta_{E}}\left\|e_{h}\right\|_{\text {supg }, E} \\
& \lesssim \tau_{E}^{1 / 2} h_{E}^{s} \frac{|f \boldsymbol{\beta}|_{s, E}}{\beta_{E}}\left\|e_{h}\right\|_{\mathrm{supg}, E} \lesssim \tau_{E}^{1 / 2} \frac{\|\boldsymbol{\beta}\|_{\left[W_{\infty}^{s}(E)\right]^{2}}}{\beta_{E}} h_{E}^{s}\|f\|_{s, E}\left\|e_{h}\right\|_{\mathrm{supg}, E} .
\end{aligned}
$$

Now the thesis follows from (5.8), (5.9) and (5.10).

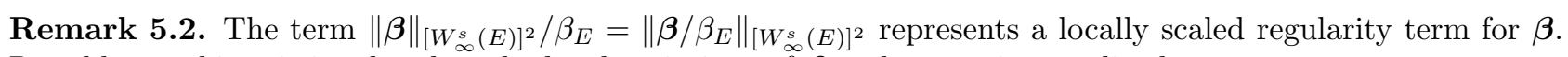
Roughly speaking, it is related to the local variations of $\boldsymbol{\beta}$ and not to its amplitude.

Lemma 5.3 (Estimate of $\left.\eta_{a}^{E}\right)$. Under the assumptions (A.1) and (A.2), the term $\eta_{a}^{E}$ can be bounded as follows $($ for $0<s \leq k)$

$$
\eta_{a}^{E} \lesssim \varepsilon^{1 / 2} h_{E}^{s}|u|_{s+1, E}\left\|e_{h}\right\|_{\text {supg, } E} .
$$

Proof. The consistency and the continuity of the form $a_{h}^{E}(\cdot, \cdot)$, Lemmas 3.1 and 4.1 easily imply

$$
\begin{aligned}
\eta_{a}^{E} & =\varepsilon a^{E}\left(u, e_{h}\right)-\varepsilon a_{h}^{E}\left(u_{\mathcal{I}}, e_{h}\right)=\varepsilon a^{E}\left(u-\Pi_{k}^{\nabla, E} u, e_{h}\right)+\varepsilon a_{h}^{E}\left(\Pi_{k}^{\nabla, E} u-u_{\mathcal{I}}, e_{h}\right) \\
& \leq \varepsilon\left(\left\|\nabla e_{\pi}\right\|_{0, E}+\left(1+\alpha^{*}\right)\left\|\nabla\left(\Pi_{k}^{\nabla, E} u-u_{\mathcal{I}}\right)\right\|_{0, E}\right)\left\|\nabla e_{h}\right\|_{0, E} \\
& \lesssim \varepsilon\left(\left\|\nabla e_{\mathcal{I}}\right\|_{0, E}+\left\|\nabla e_{\pi}\right\|_{0, E}\right)\left\|\nabla e_{h}\right\|_{0, E} \lesssim \varepsilon^{1 / 2} h_{E}^{s}|u|_{s+1, E}\left\|e_{h}\right\|_{\text {supg }, E} .
\end{aligned}
$$


Lemma 5.4 (Estimate of $\eta_{\mathcal{B}}^{E}$ ). Under the assumptions (A.1) and (A.2), the term $\eta_{\mathcal{B}}^{E}$ can be bounded as follows $($ for $0<s \leq k$ )

$$
\eta_{\mathcal{B}}^{E} \lesssim \tau_{E}^{1 / 2} \beta_{E} \frac{\|\boldsymbol{\beta}\|_{\left[W_{\infty}^{s}(E)\right]^{2}}^{2}}{\beta_{E}^{2}} h_{E}^{s}\|u\|_{s+1, E}\left\|e_{h}\right\|_{\text {supg }, E} .
$$

Proof. Using the definition of $L^{2}$-projection, simple computations yield

$$
\begin{aligned}
\eta_{\mathcal{B}}^{E}= & \tau_{E}\left(\boldsymbol{\beta} \cdot \nabla u, \boldsymbol{\beta} \cdot \nabla e_{h}\right)_{0, E}-\tau_{E}\left(\boldsymbol{\beta} \cdot \boldsymbol{\Pi}_{k-1}^{0, E} \nabla u_{\mathcal{I}}, \boldsymbol{\beta} \cdot \boldsymbol{\Pi}_{k-1}^{0, E} \nabla e_{h}\right)_{0, E} \\
& -\tau_{E} \beta_{E}^{2} \mathcal{S}^{E}\left(\left(I-\Pi_{k}^{\nabla, E}\right) u_{\mathcal{I}},\left(I-\Pi_{k}^{\nabla, E}\right) e_{h}\right) \\
= & \tau_{E}\left(\boldsymbol{\beta} \cdot \nabla u-\boldsymbol{\beta} \cdot \boldsymbol{\Pi}_{k-1}^{0, E} \nabla u_{\mathcal{I}}, \boldsymbol{\beta} \cdot \boldsymbol{\Pi}_{k-1}^{0, E} \nabla e_{h}\right)_{0, E}+\tau_{E}\left(\boldsymbol{\beta} \cdot \nabla u, \boldsymbol{\beta} \cdot\left(I-\boldsymbol{\Pi}_{k-1}^{0, E}\right) \nabla e_{h}\right)_{0, E} \\
& -\tau_{E} \beta_{E}^{2} \mathcal{S}^{E}\left(\left(I-\Pi_{k}^{\nabla, E}\right) u_{\mathcal{I}},\left(I-\Pi_{k}^{\nabla, E}\right) e_{h}\right) \\
= & \tau_{E}\left(\boldsymbol{\beta} \cdot \nabla u-\boldsymbol{\beta} \cdot \boldsymbol{\Pi}_{k-1}^{0, E} \nabla u_{\mathcal{I}}, \boldsymbol{\beta} \cdot \boldsymbol{\Pi}_{k-1}^{0, E} \nabla e_{h}\right)_{0, E} \\
& +\tau_{E}\left(\left(I-\boldsymbol{\Pi}_{k-1}^{0, E}\right) \boldsymbol{\beta} \boldsymbol{\beta}^{\mathrm{T}} \nabla u,\left(I-\boldsymbol{\Pi}_{k-1}^{0, E}\right) \nabla e_{h}\right)_{0, E} \\
& -\tau_{E} \beta_{E}^{2} \mathcal{S}^{E}\left(\left(I-\Pi_{k}^{\nabla, E}\right) u_{\mathcal{I}},\left(I-\Pi_{k}^{\nabla, E}\right) e_{h}\right) \\
= & : \eta_{\mathcal{B}, 1}^{E}+\eta_{\mathcal{B}, 2}^{E}+\eta_{\mathcal{B}, 3}^{E} .
\end{aligned}
$$

We analyse separately each term in the sum. The term $\eta_{\mathcal{B}, 1}^{E}$ is bounded using (5.6) and the continuity of $\boldsymbol{\Pi}_{k-1}^{0, E}$ with respect to the $L^{2}$-norm, Lemmas 3.1 and 4.1 :

$$
\begin{aligned}
\eta_{\mathcal{B}, 1}^{E} & \leq \tau_{E}\left\|\boldsymbol{\beta} \cdot \nabla u-\boldsymbol{\beta} \cdot \boldsymbol{\Pi}_{k-1}^{0, E} \nabla u_{\mathcal{I}}\right\|_{0, E}\left\|\boldsymbol{\beta} \cdot \boldsymbol{\Pi}_{k-1}^{0, E} \nabla e_{h}\right\|_{0, E} \\
& \leq \tau_{E}^{1 / 2} \beta_{E}\left\|\nabla u-\boldsymbol{\Pi}_{k-1}^{0, E} \nabla u_{\mathcal{I}}\right\|_{0, E}\left\|e_{h}\right\|_{\text {supg, } E} \\
& \leq \tau_{E}^{1 / 2} \beta_{E}\left(\left\|\left(I-\boldsymbol{\Pi}_{k-1}^{0, E}\right) \nabla u\right\|_{0, E}+\left\|\boldsymbol{\Pi}_{k-1}^{0, E} \nabla\left(u-u_{\mathcal{I}}\right)\right\|_{0, E}\right)\left\|e_{h}\right\|_{\text {supg }, E} \\
& \leq \tau_{E}^{1 / 2} \beta_{E}\left(\left\|\nabla e_{\pi}\right\|_{0, E}+\left\|\nabla e_{\mathcal{I}}\right\|_{0, E}\right)\left\|e_{h}\right\|_{\text {supg }, E} \\
& \lesssim \tau_{E}^{1 / 2} \beta_{E} h_{E}^{s}|u|_{s+1, E}\left\|e_{h}\right\|_{\text {supg }, E}
\end{aligned}
$$

For the second term $\eta_{\mathcal{B}, 2}^{E}$ using again (5.6) and Lemma 3.1 we infer

$$
\begin{aligned}
\eta_{\mathcal{B}, 2}^{E} & \leq \tau_{E}\left\|\left(I-\boldsymbol{\Pi}_{k-1}^{0, E}\right) \boldsymbol{\beta} \boldsymbol{\beta}^{\mathrm{T}} \nabla u\right\|_{0, E}\left\|\left(I-\boldsymbol{\Pi}_{k-1}^{0, E}\right) \nabla e_{h}\right\|_{0, E} \\
& \leq \tau_{E}^{1 / 2} \beta_{E} \frac{\left\|\left(I-\boldsymbol{\Pi}_{k-1}^{0, E}\right) \boldsymbol{\beta} \boldsymbol{\beta}^{\mathrm{T}} \nabla u\right\|_{0, E}}{\beta_{E}^{2}}\left\|e_{h}\right\|_{\text {supg }, E} \\
& \lesssim \tau_{E}^{1 / 2} \beta_{E} h_{E}^{s} \frac{\left|\boldsymbol{\beta} \boldsymbol{\beta}^{\mathrm{T}} \nabla u\right|_{s, E}}{\beta_{E}^{2}}\left\|e_{h}\right\|_{\mathrm{supg}, E} \lesssim \tau_{E}^{1 / 2} \beta_{E} \frac{\|\boldsymbol{\beta}\|_{\left[W_{\infty}^{s}(E)\right]^{2}}^{2}}{\beta_{E}^{2}} h_{E}^{s}\|u\|_{s+1, E}\left\|e_{h}\right\|_{\text {supg }, E} .
\end{aligned}
$$

Finally for the last term in (5.11), employing (4.4), the stability of the $H^{1}$-seminorm projection with respect to the $H^{1}$-seminorm, Lemma 3.1 and 4.1 we get

$$
\begin{aligned}
\eta_{\mathcal{B}, 3}^{E} & =-\tau_{E} \beta_{E}^{2} \mathcal{S}^{E}\left(\left(I-\Pi_{k}^{\nabla, E}\right) u_{\mathcal{I}},\left(I-\Pi_{k}^{\nabla, E}\right) e_{h}\right) \\
& \leq \alpha^{*} \tau_{E} \beta_{E}^{2}\left\|\nabla\left(I-\Pi_{k}^{\nabla, E}\right) u_{\mathcal{I}}\right\|_{0, E}\left\|\nabla\left(I-\Pi_{k}^{\nabla, E}\right) e_{h}\right\|_{0, E} \\
& \leq \alpha^{*} \tau_{E}^{1 / 2} \beta_{E}\left(\left\|\nabla e_{\mathcal{I}}\right\|_{0, E}+\left\|\nabla e_{\pi}\right\|_{0, E}\right)\left\|e_{h}\right\|_{\text {supg }, E} \\
& \lesssim \tau_{E}^{1 / 2} \beta_{E} h_{E}^{s}|u|_{s+1, E}\left\|e_{h}\right\|_{\text {supg }, E} .
\end{aligned}
$$

The thesis now follows by collecting (5.12), (5.13) and (5.14) in (5.11). 
Lemma 5.5 (Estimate of $\left.\eta_{\mathcal{L}}^{E}\right)$. Under the assumptions (A.1) and (A.2), the term $\eta_{\mathcal{L}}^{E}$ can be bounded as follows $($ for $0<s \leq k$ )

$$
\eta_{\mathcal{L}}^{E} \lesssim \tau_{E}^{1 / 2} \varepsilon \frac{\|\boldsymbol{\beta}\|_{\left[W_{\infty}^{\max \{s-1,0\}}(E)\right]^{2}}}{\beta_{E}} h_{E}^{\max \{s-1,0\}}\|u\|_{s+1, E}\left\|e_{h}\right\|_{\text {supg, }, E}
$$

Proof. By definition of $L^{2}$-orthogonal projection we infer

$$
\begin{aligned}
\eta_{\mathcal{L}}^{E} & =\tau_{E} \varepsilon\left(\operatorname{div} \boldsymbol{\Pi}_{k-1}^{0, E} \nabla u_{\mathcal{I}}, \boldsymbol{\beta} \cdot \boldsymbol{\Pi}_{k-1}^{0, E} \nabla e_{h}\right)_{0, E}-\tau_{E} \varepsilon\left(\Delta u, \boldsymbol{\beta} \cdot \nabla e_{h}\right)_{0, E} \\
& =\tau_{E} \varepsilon\left(\operatorname{div}\left(\boldsymbol{\Pi}_{k-1}^{0, E} \nabla u_{\mathcal{I}}-\nabla u\right), \boldsymbol{\beta} \cdot \boldsymbol{\Pi}_{k-1}^{0, E} \nabla e_{h}\right)_{0, E}-\tau_{E} \varepsilon\left(\Delta u, \boldsymbol{\beta} \cdot\left(I-\boldsymbol{\Pi}_{k-1}^{0, E}\right) \nabla e_{h}\right)_{0, E} \\
& =\tau_{E} \varepsilon\left(\operatorname{div}\left(\boldsymbol{\Pi}_{k-1}^{0, E} \nabla u_{\mathcal{I}}-\nabla u\right), \boldsymbol{\beta} \cdot \boldsymbol{\Pi}_{k-1}^{0, E} \nabla e_{h}\right)_{0, E}-\tau_{E} \varepsilon\left(\left(I-\boldsymbol{\Pi}_{k-1}^{0, E}\right) \Delta u \boldsymbol{\beta},\left(I-\boldsymbol{\Pi}_{k-1}^{0, E}\right) \nabla e_{h}\right)_{0, E} \\
& =: \eta_{\mathcal{L}, 1}^{E}+\eta_{\mathcal{L}, 2}^{E} .
\end{aligned}
$$

The term $\eta_{\mathcal{L}, 1}^{E}$, employing Lemmas 3.2, 3.1, and 4.1 is estimated as follows

$$
\begin{aligned}
\eta_{\mathcal{L}, 1}^{E} & \leq \tau_{E} \varepsilon\left\|\operatorname{div}\left(\nabla u-\boldsymbol{\Pi}_{k-1}^{0, E} \nabla u_{\mathcal{I}}\right)\right\|_{0, E}\left\|\boldsymbol{\beta} \cdot \boldsymbol{\Pi}_{k-1}^{0, E} \nabla e_{h}\right\|_{0, E} \\
& \leq \tau_{E}^{1 / 2} \varepsilon\left\|\operatorname{div}\left(\nabla u-\boldsymbol{\Pi}_{k-1}^{0, E} \nabla u_{\mathcal{I}}\right)\right\|_{0, E}\left\|e_{h}\right\|_{\mathrm{supg}, E} \\
& \leq \tau_{E}^{1 / 2} \varepsilon\left(\left\|\operatorname{div}\left(\nabla u-\boldsymbol{\Pi}_{k-1}^{0, E} \nabla u\right)\right\|_{0, E}+\left\|\operatorname{div} \boldsymbol{\Pi}_{k-1}^{0, E}\left(\nabla u-\nabla u_{\mathcal{I}}\right)\right\|_{0, E}\right)\left\|e_{h}\right\|_{\text {supg }, E} \\
& \leq \tau_{E}^{1 / 2} \varepsilon\left(\left|\left(I-\boldsymbol{\Pi}_{k-1}^{0, E}\right) \nabla u\right|_{1, E}+h_{E}^{-1} \gamma_{E}^{1 / 2}\left\|\nabla e_{\mathcal{I}}\right\|_{0, E}\right)\left\|e_{h}\right\|_{\text {supg }, E} \\
& \lesssim \tau_{E}^{1 / 2} \varepsilon h_{E}^{s-1}|u|_{s+1, E}\left\|e_{h}\right\|_{\text {supg, }, E} .
\end{aligned}
$$

The second term in (5.15), recalling (5.6), can be easily bounded as follows

$$
\begin{aligned}
\eta_{\mathcal{L}, 2}^{E} & \leq \tau_{E} \varepsilon\left\|\left(I-\boldsymbol{\Pi}_{k-1}^{0, E}\right) \Delta u \boldsymbol{\beta}\right\|_{0, E}\left\|\left(I-\boldsymbol{\Pi}_{k-1}^{0, E}\right) \nabla e_{h}\right\|_{0, E} \leq \tau_{E}^{1 / 2} \varepsilon \frac{\left\|\left(I-\boldsymbol{\Pi}_{k-1}^{0, E}\right) \Delta u \boldsymbol{\beta}\right\|_{0, E}}{\beta_{E}}\left\|e_{h}\right\|_{\text {supg, } E} \\
& \lesssim \tau_{E}^{1 / 2} \varepsilon \frac{|\Delta u \boldsymbol{\beta}|_{\vartheta, E}}{\beta_{E}} h_{E}^{\vartheta}\left\|e_{h}\right\|_{\mathrm{supg}, E} \lesssim \tau_{E}^{1 / 2} \varepsilon \frac{\|\boldsymbol{\beta}\|_{\left[W_{\infty}^{\vartheta}(E)\right]^{2}}}{\beta_{E}^{\vartheta}} h_{E}\|u\|_{s+1, E}\left\|e_{h}\right\|_{\mathrm{supg}, E} .
\end{aligned}
$$

where $\vartheta=\max \{s-1,0\}$. Collecting (5.16) and (5.17) in (5.15) we get the thesis.

The last and most challenging step in the analysis consists in estimating the term $\eta_{b}^{E}$ in (5.7) for both $b_{o, h}^{E}$ and $b_{\partial, h}^{E}$ (that we denote respectively by $\eta_{b, o}^{E}$ and $\eta_{b, \partial}^{E}$ ), see also Remark 5.3.

Lemma 5.6 (Estimate of $\left.\eta_{b, o}^{E}\right)$. Let $b_{o, h}^{E}(\cdot, \cdot)$ be the bilinear form in (4.5). Then under assumptions (A.1) and (A.2), the term $\eta_{b, o}^{E}$ can be bounded as follows (for $0<s \leq k$ )

$$
\begin{array}{r}
\eta_{b, o}^{E} \lesssim\left(\sigma_{E} \frac{\|\boldsymbol{\beta}\|_{\left[W_{\infty}^{s+1}(E)\right]^{2}}}{\beta_{E}} h_{E}^{s+1}\|u\|_{s+1, E}+\frac{1}{\varepsilon^{1 / 2}}|\boldsymbol{\beta}|_{\left[W_{\infty}^{1}(E)\right]^{2}} h_{E}^{s+2}|u|_{s+1, E}\right)\left\|e_{h}\right\|_{\text {supg }, E}+ \\
+|\boldsymbol{\beta}|_{\left[W_{\infty}^{s+1}(E)\right]^{2}} h_{E}^{2 s+1}|u|_{s+1, E}\left\|e_{h}\right\|_{0, E}+\int_{\partial E}\left(\boldsymbol{\beta} \cdot \boldsymbol{n}^{E}\right) e_{\mathcal{I}} e_{h} \mathrm{~d} s
\end{array}
$$

where for any $E \in \Omega_{h}$

$$
\sigma_{E}=\min \left\{\frac{\beta_{E}}{\varepsilon^{1 / 2}}, \frac{1}{\tau_{E}^{1 / 2}}\right\}=\beta_{E} \lambda_{E} .
$$


Proof. By the definition of the skew symmetric forms (2.4) and (4.7) we need to estimate the terms

$$
\begin{aligned}
\eta_{b, A}^{E} & :=\left(\boldsymbol{\beta} \cdot \nabla u, e_{h}\right)_{E}-\left(\boldsymbol{\beta} \cdot \boldsymbol{\Pi}_{k}^{0, E} \nabla u_{\mathcal{I}}, \Pi_{k}^{0, E} e_{h}\right)_{E}, \\
\eta_{b, B}^{E} & :=\left(\Pi_{k}^{0, E} u_{\mathcal{I}}, \boldsymbol{\beta} \cdot \boldsymbol{\Pi}_{k}^{0, E} \nabla e_{h}\right)_{E}-\left(u, \boldsymbol{\beta} \cdot \nabla e_{h}\right)_{E} .
\end{aligned}
$$

Using usual computations we infer

$$
\begin{aligned}
& \eta_{b, A}^{E}=\left(\boldsymbol{\beta} \cdot \nabla u, e_{h}\right)_{E}-\left(\boldsymbol{\beta} \cdot \boldsymbol{\Pi}_{k}^{0, E} \nabla u_{\mathcal{I}}, \Pi_{k}^{0, E} e_{h}\right)_{E} \\
& =\left(\boldsymbol{\beta} \cdot \nabla u, e_{h}\right)_{E}-\left(\boldsymbol{\beta} \cdot \nabla u_{\mathcal{I}}, \Pi_{k}^{0, E} e_{h}\right)_{E}+\left(\boldsymbol{\beta} \cdot\left(I-\boldsymbol{\Pi}_{k}^{0, E}\right) \nabla u_{\mathcal{I}}, \Pi_{k}^{0, E} e_{h}\right)_{E} \\
& =\left(\boldsymbol{\beta} \cdot \nabla\left(u-u_{\mathcal{I}}\right), e_{h}\right)_{E}+\left(\boldsymbol{\beta} \cdot \nabla u_{\mathcal{I}},\left(I-\Pi_{k}^{0, E}\right) e_{h}\right)_{E}+\left(\left(I-\Pi_{k}^{0, E}\right) \nabla u_{\mathcal{I}}, \boldsymbol{\beta} \Pi_{k}^{0, E} e_{h}\right)_{E} \\
& =\left(\boldsymbol{\beta} \cdot \nabla e_{\mathcal{I}}, e_{h}\right)_{E}+\left(\left(I-\Pi_{k}^{0, E}\right) \boldsymbol{\beta} \cdot \nabla u_{\mathcal{I}},\left(I-\Pi_{k}^{0, E}\right) e_{h}\right)_{E}+ \\
& +\left(\left(I-\boldsymbol{\Pi}_{k}^{0, E}\right) \nabla u_{\mathcal{I}}, \boldsymbol{\beta}\left(\Pi_{k}^{0, E} e_{h}-\Pi_{0}^{0, E} e_{h}\right)\right)_{E}+\left(\left(I-\boldsymbol{\Pi}_{k}^{0, E}\right) \nabla u_{\mathcal{I}}, \boldsymbol{\beta} \Pi_{0}^{0, E} e_{h}\right)_{E} \\
& =: \eta_{b, 1}^{E}+\eta_{b, 2}^{E}+\eta_{b, 3}^{E}+\eta_{b, 4}^{E} \text {, } \\
& \eta_{b, B}^{E}=\left(\Pi_{k}^{0, E} u_{\mathcal{I}}, \boldsymbol{\beta} \cdot \boldsymbol{\Pi}_{k}^{0, E} \nabla e_{h}\right)_{E}-\left(u, \boldsymbol{\beta} \cdot \nabla e_{h}\right)_{E} \\
& =\left(\Pi_{k}^{0, E} u_{\mathcal{I}}-u, \boldsymbol{\beta} \cdot \boldsymbol{\Pi}_{k}^{0, E} \nabla e_{h}\right)_{E}+\left(u, \boldsymbol{\beta} \cdot\left(\boldsymbol{\Pi}_{k}^{0, E}-I\right) \nabla e_{h}\right)_{E} \\
& =\left(\Pi_{k}^{0, E} u_{\mathcal{I}}-u, \boldsymbol{\beta} \cdot \boldsymbol{\Pi}_{k}^{0, E} \nabla e_{h}\right)_{E}+\left(\left(I-\boldsymbol{\Pi}_{k}^{0, E}\right) \boldsymbol{\beta} u,\left(\boldsymbol{\Pi}_{k}^{0, E}-I\right) \nabla e_{h}\right)_{E} \\
& =: \eta_{b, 5}^{E}+\eta_{b, 6}^{E} \text {, }
\end{aligned}
$$

yielding the following expression for $\eta_{b, o}^{E}$

$$
2 \eta_{b, o}^{E}=\eta_{b, 1}^{E}+\eta_{b, 2}^{E}+\eta_{b, 3}^{E}+\eta_{b, 4}^{E}+\eta_{b, 5}^{E}+\eta_{b, 6}^{E}
$$

We now analyse each term $\eta_{b, i}^{E}$ for $i=1, \ldots, 6$ in the sum above.

- $\eta_{b, 1}^{E}$ : using an integration by parts, bound (5.6) and the definition of $\|\cdot\|_{\mathrm{supg}, E}$ we infer

$$
\begin{aligned}
\eta_{b, 1}^{E} & =\left(\boldsymbol{\beta} \cdot \nabla e_{\mathcal{I}}, e_{h}\right)_{E}=-\left(e_{\mathcal{I}}, \boldsymbol{\beta} \cdot \nabla e_{h}\right)_{E}+\int_{\partial E}\left(\boldsymbol{\beta} \cdot \boldsymbol{n}^{E}\right) e_{\mathcal{I}} e_{h} \mathrm{~d} s \\
& \leq\left\|e_{\mathcal{I}}\right\|_{0, E}\left\|\boldsymbol{\beta} \cdot \nabla e_{h}\right\|_{0, E}+\int_{\partial E}\left(\boldsymbol{\beta} \cdot \boldsymbol{n}^{E}\right) e_{\mathcal{I}} e_{h} \mathrm{~d} s \\
& \leq\left\|e_{\mathcal{I}}\right\|_{0, E}\left(\left\|\boldsymbol{\beta} \cdot \boldsymbol{\Pi}_{k-1}^{0, E} \nabla e_{h}\right\|_{0, E}+\beta_{E}\left\|\nabla\left(I-\Pi_{k}^{\nabla, E}\right) e_{h}\right\|_{0, E}\right)+\int_{\partial E}\left(\boldsymbol{\beta} \cdot \boldsymbol{n}^{E}\right) e_{\mathcal{I}} e_{h} \mathrm{~d} s \\
& \lesssim \min \left\{\frac{\beta_{E}}{\varepsilon^{1 / 2}}, \frac{1}{\tau_{E}^{1 / 2}}\right\}\left\|e_{\mathcal{I}}\right\|_{0, E}\left\|e_{h}\right\|_{\text {supg }, E}+\int_{\partial E}\left(\boldsymbol{\beta} \cdot \boldsymbol{n}^{E}\right) e_{\mathcal{I}} e_{h} \mathrm{~d} s \\
s & \lesssim \sigma_{E} h_{E}^{s+1}|u|_{s+1, E}\left\|e_{h}\right\|_{\text {supg }, E}+\int_{\partial E}\left(\boldsymbol{\beta} \cdot \boldsymbol{n}^{E}\right) e_{\mathcal{I}} e_{h} \mathrm{~d} s .
\end{aligned}
$$


$-\eta_{b, 2}^{E}$ : a scaled Poincaré inequality and the definition of $L^{2}$-projection imply

$$
\begin{aligned}
\eta_{b, 2}^{E} & =\left(\left(I-\Pi_{k}^{0, E}\right) \boldsymbol{\beta} \cdot \nabla u_{\mathcal{I}},\left(I-\Pi_{k}^{0, E}\right) e_{h}\right)_{E} \\
& =\left(\left(I-\Pi_{k}^{0, E}\right) \boldsymbol{\beta} \cdot \nabla u,\left(I-\Pi_{k}^{0, E}\right) e_{h}\right)_{E}-\left(\left(I-\Pi_{k}^{0, E}\right) \boldsymbol{\beta} \cdot \nabla e_{\mathcal{I}},\left(I-\Pi_{k}^{0, E}\right) e_{h}\right)_{E} \\
& \leq\left(\left\|\left(I-\Pi_{k}^{0, E}\right) \boldsymbol{\beta} \cdot \nabla u\right\|_{0, E}+\left\|\boldsymbol{\beta} \cdot \nabla e_{\mathcal{I}}\right\|_{0, E}\right)\left\|\left(I-\Pi_{k}^{0, E}\right) e_{h}\right\|_{0, E} \\
& \leq\left(\left\|\left(I-\Pi_{k}^{0, E}\right) \boldsymbol{\beta} \cdot \nabla u\right\|_{0, E}+\left\|\boldsymbol{\beta} \cdot \nabla e_{\mathcal{I}}\right\|_{0, E}\right)\left\|\left(I-\Pi_{k}^{\nabla, E}\right) e_{h}\right\|_{0, E} \\
& \lesssim \min \left\{\frac{\beta_{E}}{\varepsilon^{1 / 2}}, \frac{1}{\tau_{E}^{1 / 2}}\right\} h_{E}\left(\frac{\left\|\left(I-\Pi_{k}^{0, E}\right) \boldsymbol{\beta} \cdot \nabla u\right\|_{0, E}}{\beta_{E}}+\frac{\left\|\boldsymbol{\beta} \cdot \nabla e_{\mathcal{I}}\right\|_{0, E}}{\beta_{E}}\right)\left\|e_{h}\right\|_{\mathrm{supg}, E} \\
& \lesssim \sigma_{E}\left(\frac{|\boldsymbol{\beta} \cdot \nabla u|_{s, E}}{\beta_{E}}+|u|_{s+1, E}\right) h_{E}^{s+1}\left\|e_{h}\right\|_{\mathrm{supg}, E} \\
& \lesssim \sigma_{E}\left(\frac{\|\boldsymbol{\beta}\|_{\left[W_{\infty}^{s}(E)\right]^{2}}}{\beta_{E}}+1\right) h_{E}^{s+1}\|u\|_{s+1, E}\left\|e_{h}\right\|_{\mathrm{supg}, E} .
\end{aligned}
$$

- $\eta_{b, 3}^{E}$ : from the definition of $L^{2}$-projection, the Poincaré inequality and Lemma 3.1, we infer

$$
\begin{aligned}
\eta_{b, 3}^{E} & =\left(\left(I-\boldsymbol{\Pi}_{k}^{0, E}\right) \nabla u_{\mathcal{I}}, \boldsymbol{\beta}\left(\Pi_{k}^{0, E} e_{h}-\Pi_{0}^{0, E} e_{h}\right)\right)_{E} \\
& =\left(\left(I-\boldsymbol{\Pi}_{k}^{0, E}\right) \nabla u_{\mathcal{I}},\left(\boldsymbol{\beta}-\boldsymbol{\Pi}_{0}^{0, E} \boldsymbol{\beta}\right)\left(\Pi_{k}^{0, E} e_{h}-\Pi_{0}^{0, E} e_{h}\right)\right)_{E} \\
& \leq\left\|\left(I-\boldsymbol{\Pi}_{k}^{0, E}\right) \nabla u_{\mathcal{I}}\right\|_{0, E}\left\|\left(I-\boldsymbol{\Pi}_{0}^{0, E}\right) \boldsymbol{\beta}\right\|_{L^{\infty}}\left\|\left(\Pi_{k}^{0, E}-\Pi_{0}^{0, E}\right) e_{h}\right\|_{0, E} \\
& \leq\left\|\left(I-\boldsymbol{\Pi}_{k-1}^{0, E}\right) \nabla u_{\mathcal{I}}\right\|_{0, E}\left\|\left(I-\boldsymbol{\Pi}_{0}^{0, E}\right) \boldsymbol{\beta}\right\|_{L^{\infty}}\left\|\left(I-\Pi_{0}^{0, E}\right) e_{h}\right\|_{0, E} \\
& \lesssim \frac{h_{E}}{\varepsilon^{1 / 2}}\left(\left\|\nabla e_{\mathcal{I}}\right\|_{0, E}+\left\|\nabla e_{\pi}\right\|_{0, E}\right)\left\|\left(I-\boldsymbol{\Pi}_{0}^{0, E}\right) \boldsymbol{\beta}\right\|_{L^{\infty}}\left\|e_{h}\right\|_{\text {supg }, E} \\
& \lesssim \frac{1}{\varepsilon^{1 / 2}}|\boldsymbol{\beta}|_{\left[W_{\infty}^{1}(E)\right]^{2}} h_{E}^{s+2}|u|_{s+1, E}\left\|e_{h}\right\|_{\text {supg }, E} .
\end{aligned}
$$

$-\eta_{b, 4}^{E}$ : using similar computations of the previous item we obtain

$$
\begin{aligned}
\eta_{b, 4}^{E} & =\left(\left(I-\boldsymbol{\Pi}_{k}^{0, E}\right) \nabla u_{\mathcal{I}}, \boldsymbol{\beta} \Pi_{0}^{0, E} e_{h}\right)_{E} \\
& =\left(\left(I-\boldsymbol{\Pi}_{k}^{0, E}\right) \nabla u_{\mathcal{I}},\left(\boldsymbol{\beta}-\boldsymbol{\Pi}_{k}^{0, E} \boldsymbol{\beta}\right) \Pi_{0}^{0, E} e_{h}\right)_{E} \\
& \leq\left\|\left(I-\boldsymbol{\Pi}_{k}^{0, E}\right) \nabla u_{\mathcal{I}}\right\|_{0, E}\left\|\left(I-\boldsymbol{\Pi}_{0}^{k, E}\right) \boldsymbol{\beta}\right\|_{L^{\infty}}\left\|\Pi_{0}^{0, E} e_{h}\right\|_{0, E} \\
& \leq\left(\left\|\nabla e_{\mathcal{I}}\right\|_{0, E}+\left\|\nabla e_{\pi}\right\|_{0, E}\right)\left\|\left(I-\boldsymbol{\Pi}_{0}^{k, E}\right) \boldsymbol{\beta}\right\|_{L^{\infty}}\left\|e_{h}\right\|_{0, E} \\
& \lesssim|\boldsymbol{\beta}|_{\left[W_{\infty}^{s+1}(E)\right]^{2}} h_{E}^{2 s+1}|u|_{s+1, E}\left\|e_{h}\right\|_{0, E} .
\end{aligned}
$$

- $\eta_{b, 5}^{E}$ : exploiting the property of $L^{2}$-projection and bound (5.6) we get

$$
\begin{aligned}
\eta_{b, 5}^{E} & =\left(\Pi_{k}^{0, E} u_{\mathcal{I}}-u, \boldsymbol{\beta} \cdot \boldsymbol{\Pi}_{k}^{0, E} \nabla e_{h}\right)_{E} \\
& =\left(\Pi_{k}^{0, E} u_{\mathcal{I}}-u, \boldsymbol{\beta} \cdot \boldsymbol{\Pi}_{k-1}^{0, E} \nabla e_{h}\right)_{E}+\left(\Pi_{k}^{0, E} u_{\mathcal{I}}-u, \boldsymbol{\beta} \cdot\left(\boldsymbol{\Pi}_{k}^{0, E}-\boldsymbol{\Pi}_{k-1}^{0, E}\right) \nabla e_{h}\right)_{E} \\
& \leq\left\|\Pi_{k}^{0, E} u_{\mathcal{I}}-u\right\|_{0, E}\left(\left\|\boldsymbol{\beta} \cdot \boldsymbol{\Pi}_{k-1}^{0, E} \nabla e_{h}\right\|_{0, E}+\beta_{E}\left\|\left(\boldsymbol{\Pi}_{k}^{0, E}-\boldsymbol{\Pi}_{k-1}^{0, E}\right) \nabla e_{h}\right\|_{0, E}\right) \\
& \leq\left(\left\|\left(I-\Pi_{k}^{0, E}\right) u\right\|_{0, E}+\left\|e_{\mathcal{I}}\right\|_{0, E}\right)\left(\left\|\boldsymbol{\beta} \cdot \boldsymbol{\Pi}_{k-1}^{0, E} \nabla e_{h}\right\|_{0, E}+\beta_{E}\left\|\nabla\left(I-\Pi_{k}^{\nabla, E}\right) e_{h}\right\|_{0, E}\right) \\
& \leq \min \left\{\frac{\beta_{E}}{\varepsilon^{1 / 2}}, \frac{1}{\tau_{E}^{1 / 2}}\right\}\left(\left\|\left(I-\Pi_{k}^{0, E}\right) u\right\|_{0, E}+\left\|e_{\mathcal{I}}\right\|_{0, E}\right)\left\|e_{h}\right\|_{\operatorname{supg}, E} \\
& \lesssim \sigma_{E} h_{E}^{s+1}|u|_{s+1, E}\left\|e_{h}\right\|_{\text {supg }, E} .
\end{aligned}
$$


$-\eta_{b, 6}^{E}$ : using similar computations of the previous item we have

$$
\begin{aligned}
\eta_{b, 6}^{E} & =\left(\left(I-\boldsymbol{\Pi}_{k}^{0, E}\right) \boldsymbol{\beta} u,\left(\boldsymbol{\Pi}_{k}^{0, E}-I\right) \nabla e_{h}\right)_{E} \\
& \leq\left\|\left(I-\boldsymbol{\Pi}_{k}^{0, E}\right) \boldsymbol{\beta} u\right\|_{0, E}\left\|\left(\boldsymbol{\Pi}_{k}^{0, E}-I\right) \nabla e_{h}\right\|_{0, E} \leq\left\|\left(I-\boldsymbol{\Pi}_{k}^{0, E}\right) \boldsymbol{\beta} u\right\|_{0, E}\left\|\nabla\left(I-\Pi_{k}^{\nabla, E}\right) e_{h}\right\|_{0, E} \\
& \leq \min \left\{\frac{\beta_{E}}{\varepsilon^{1 / 2}}, \frac{1}{\tau_{E}^{1 / 2}}\right\} \frac{\left\|\left(I-\boldsymbol{\Pi}_{k}^{0, E}\right) \boldsymbol{\beta} u\right\|_{0, E}}{\beta_{E}}\left\|e_{h}\right\|_{\text {supg }, E} \\
& \lesssim \sigma_{E} \frac{|\boldsymbol{\beta} u|_{s+1, E}}{\beta_{E}} h_{E}^{s+1}\left\|e_{h}\right\|_{\text {supg }, E} \\
& \lesssim \sigma_{E} \frac{\|\boldsymbol{\beta}\|_{\left[W_{\infty}^{s+1}(E)\right]^{2}}}{\beta_{E}} h_{E}^{s+1}\|u\|_{s+1, E}\left\|e_{h}\right\|_{\text {supg }, E} .
\end{aligned}
$$

The thesis now follows gathering (5.21)-(5.26) in (5.20).

Lemma 5.7 (Estimate of $\left.\eta_{b, \partial}^{E}\right)$. Let $b_{\partial, h}^{E}(\cdot, \cdot)$ be the bilinear form in (4.6). Then under assumptions (A.1) and (A.2), the term $\eta_{b, \partial}^{E}$ can be bounded as follows

$$
\eta_{b, \partial}^{E} \lesssim \sigma_{E} \frac{\|\boldsymbol{\beta}\|_{\left[W_{\infty}^{s+1}(E)\right]^{2}}}{\beta_{E}} h_{E}^{s+1}\|u\|_{s+1, E}\left\|e_{h}\right\|_{\text {supg }, E}+\int_{\partial E}\left(\boldsymbol{\beta} \cdot \boldsymbol{n}^{E}\right) e_{\mathcal{I}} e_{h} \mathrm{~d} s
$$

where $\sigma_{E}$ is defined in (5.19).

Proof. Recalling definition (4.6) we need to estimate the terms

$$
\begin{aligned}
\eta_{b, A}^{E} & :=\left(\boldsymbol{\beta} \cdot \nabla u, e_{h}\right)_{E}-\left(\boldsymbol{\beta} \cdot \nabla \Pi_{k}^{0, E} u_{\mathcal{I}}, \Pi_{k}^{0, E} e_{h}\right)_{E}-\int_{\partial E}\left(\boldsymbol{\beta} \cdot \boldsymbol{n}^{E}\right)\left(I-\Pi_{k}^{0, E}\right) u_{\mathcal{I}} \Pi_{k}^{0, E} e_{h} \mathrm{~d} s \\
\eta_{b, B}^{E} & :=\left(\Pi_{k}^{0, E} u_{\mathcal{I}}, \boldsymbol{\beta} \cdot \nabla \Pi_{k}^{0, E} e_{h}\right)_{E}-\left(u, \boldsymbol{\beta} \cdot \nabla e_{h}\right)_{E}+\int_{\partial E}\left(\boldsymbol{\beta} \cdot \boldsymbol{n}^{E}\right)\left(I-\Pi_{k}^{0, E}\right) e_{h} \Pi_{k}^{0, E} u_{\mathcal{I}} \mathrm{d} s .
\end{aligned}
$$

By integration by parts we have

$$
\begin{aligned}
\eta_{b, A}^{E}= & \left(\boldsymbol{\beta} \cdot \nabla u,\left(I-\Pi_{k}^{0, E}\right) e_{h}\right)_{E}+\left(\boldsymbol{\beta} \cdot \nabla\left(u-\Pi_{k}^{0, E} u_{\mathcal{I}}\right), \Pi_{k}^{0, E} e_{h}\right)_{E} \\
& \quad-\int_{\partial E}\left(\boldsymbol{\beta} \cdot \boldsymbol{n}^{E}\right)\left(I-\Pi_{k}^{0, E}\right) u_{\mathcal{I}} \Pi_{k}^{0, E} e_{h} \mathrm{~d} s \\
= & \left(\boldsymbol{\beta} \cdot \nabla u,\left(I-\Pi_{k}^{0, E}\right) e_{h}\right)_{E}-\left(u-\Pi_{k}^{0, E} u_{\mathcal{I}}, \boldsymbol{\beta} \cdot \nabla \Pi_{k}^{0, E} e_{h}\right)_{E} \\
& \quad+\int_{\partial E}\left(\boldsymbol{\beta} \cdot \boldsymbol{n}^{E}\right)\left(u-u_{\mathcal{I}}\right) \Pi_{k}^{0, E} e_{h} \mathrm{~d} s \\
= & \left(\left(I-\Pi_{k}^{0, E}\right) \boldsymbol{\beta} \cdot \nabla u,\left(I-\Pi_{k}^{0, E}\right) e_{h}\right)_{E}+\left(\Pi_{k}^{0, E} u_{\mathcal{I}}-u, \boldsymbol{\beta} \cdot \nabla \Pi_{k}^{0, E} e_{h}\right)_{E} \\
& \quad+\int_{\partial E}\left(\boldsymbol{\beta} \cdot \boldsymbol{n}^{E}\right) e_{\mathcal{I}} \Pi_{k}^{0, E} e_{h} \mathrm{~d} s \\
=: & \eta_{b, 1}^{E}+\eta_{b, 2}^{E}+\eta_{b, 3}^{E},
\end{aligned}
$$




$$
\begin{aligned}
\eta_{b, B}^{E}= & \left(\Pi_{k}^{0, E} u_{\mathcal{I}}-u, \boldsymbol{\beta} \cdot \nabla \Pi_{k}^{0, E} e_{h}\right)_{E}-\left(u, \boldsymbol{\beta} \cdot \nabla\left(I-\Pi_{k}^{0, E}\right) e_{h}\right)_{E} \\
& \quad+\int_{\partial E}\left(\boldsymbol{\beta} \cdot \boldsymbol{n}^{E}\right)\left(I-\Pi_{k}^{0, E}\right) e_{h} \Pi_{k}^{0, E} u_{\mathcal{I}} \mathrm{d} s \\
= & \left(\Pi_{k}^{0, E} u_{\mathcal{I}}-u, \boldsymbol{\beta} \cdot \nabla \Pi_{k}^{0, E} e_{h}\right)_{E}+\left(\boldsymbol{\beta} \cdot \nabla u,\left(I-\Pi_{k}^{0, E}\right) e_{h}\right)_{E} \\
& \quad+\int_{\partial E}\left(\boldsymbol{\beta} \cdot \boldsymbol{n}^{E}\right)\left(I-\Pi_{k}^{0, E}\right) e_{h}\left(\Pi_{k}^{0, E} u_{\mathcal{I}}-u\right) \mathrm{d} s \\
= & \left(\Pi_{k}^{0, E} u_{\mathcal{I}}-u, \boldsymbol{\beta} \cdot \nabla \Pi_{k}^{0, E} e_{h}\right)_{E}+\left(\left(I-\Pi_{k}^{0, E}\right) \boldsymbol{\beta} \cdot \nabla u,\left(I-\Pi_{k}^{0, E}\right) e_{h}\right)_{E} \\
& \quad+\int_{\partial E}\left(\boldsymbol{\beta} \cdot \boldsymbol{n}^{E}\right)\left(I-\Pi_{k}^{0, E}\right) e_{h}\left(\Pi_{k}^{0, E} u_{\mathcal{I}}-u\right) \mathrm{d} s \\
= & \eta_{b, 2}^{E}+\eta_{b, 1}^{E}+\eta_{b, 4}^{E},
\end{aligned}
$$

yielding the following expression for $\eta_{b, \partial}^{E}$

$$
2 \eta_{b, 2}^{E}=2 \eta_{b, 1}^{E}+2 \eta_{b, 2}^{E}+\eta_{b, 3}^{E}+\eta_{b, 4}^{E} .
$$

We now analyse each term $\eta_{b, i}^{E}$ for $i=1, \ldots, 4$ in the sum above.

- $\eta_{b, 1}^{E}$ : using the same computations in (5.22) we infer

$$
\eta_{b, 1}^{E}=\left(\left(I-\Pi_{k}^{0, E}\right) \boldsymbol{\beta} \cdot \nabla u,\left(I-\Pi_{k}^{0, E}\right) e_{h}\right)_{E} \lesssim \sigma_{E} \frac{\|\boldsymbol{\beta}\|_{\left[W_{\infty}^{s}(E)\right]^{2}}}{\beta_{E}} h_{E}^{s+1}\|u\|_{s+1, E}\left\|e_{h}\right\|_{\text {supg, } E} .
$$

$-\eta_{b, 2}^{E}$ : exploiting the computation in (5.25) we obtain

$$
\begin{aligned}
\eta_{b, 2}^{E} & =\left(\Pi_{k}^{0, E} u_{\mathcal{I}}-u, \boldsymbol{\beta} \cdot \nabla \Pi_{k}^{0, E} e_{h}\right)_{E} \\
& =\left(\Pi_{k}^{0, E} u_{\mathcal{I}}-u, \boldsymbol{\beta} \cdot \boldsymbol{\Pi}_{k-1}^{0, E} \nabla e_{h}\right)_{E}+\left(\Pi_{k}^{0, E} u_{\mathcal{I}}-u, \boldsymbol{\beta} \cdot\left(\nabla \Pi_{k}^{0, E} e_{h}-\boldsymbol{\Pi}_{k-1}^{0, E} \nabla e_{h}\right)\right)_{E} \\
& \leq\left\|\Pi_{k}^{0, E} u_{\mathcal{I}}-u\right\|_{0, E}\left(\left\|\boldsymbol{\beta} \cdot \boldsymbol{\Pi}_{k-1}^{0, E} \nabla e_{h}\right\|_{0, E}+\beta_{E}\left\|\boldsymbol{\Pi}_{k-1}^{0, E}\left(\nabla e_{h}-\nabla \Pi_{k}^{0, E} e_{h}\right)\right\|_{0, E}\right) \\
& \leq\left(\left\|\left(I-\Pi_{k}^{0, E}\right) u\right\|_{0, E}+\left\|e_{\mathcal{I}}\right\|_{0, E}\right)\left(\left\|\boldsymbol{\beta} \cdot \boldsymbol{\Pi}_{k-1}^{0, E} \nabla e_{h}\right\|_{0, E}+\beta_{E}\left\|\nabla\left(I-\Pi_{k}^{\nabla, E}\right) \nabla e_{h}\right\|_{0, E}\right) \\
& \leq \min \left\{\frac{\beta_{E}}{\varepsilon^{1 / 2}}, \frac{1}{\tau_{E}^{1 / 2}}\right\}\left(\left\|\left(I-\Pi_{k}^{0, E}\right) u\right\|_{0, E}+\left\|e_{\mathcal{I}}\right\|_{0, E}\right)\left\|e_{h}\right\|_{\operatorname{supg}, E} \\
& \lesssim \sigma_{E} h_{E}^{s+1}|u|_{s+1, E}\left\|e_{h}\right\|_{\operatorname{supg}, E} .
\end{aligned}
$$

$-\eta_{b, 3}^{E}+\eta_{b, 4}^{E}$ : we use a scaled trace inequality [18] making use of the scaled norm $\|v\|_{1, E}^{2}:=\|v\|_{L^{2}(E)}^{2}+h_{E}^{2}|v|_{H^{1}(E)}^{2}$ for all $v \in H^{1}(E)$. We obtain

$$
\begin{aligned}
\eta_{b, 3}^{E} & +\eta_{b, 4}^{E}=\int_{\partial E}\left(\boldsymbol{\beta} \cdot \boldsymbol{n}^{E}\right) e_{\mathcal{I}} \Pi_{k}^{0, E} e_{h} \mathrm{~d} s+\int_{\partial E}\left(\boldsymbol{\beta} \cdot \boldsymbol{n}^{E}\right)\left(I-\Pi_{k}^{0, E}\right) e_{h}\left(\Pi_{k}^{0, E} u_{\mathcal{I}}-u\right) \mathrm{d} s \\
& =\int_{\partial E}\left(\boldsymbol{\beta} \cdot \boldsymbol{n}^{E}\right)\left(\Pi_{k}^{0, E}-I\right) e_{h}\left(e_{\mathcal{I}}+u-\Pi_{k}^{0, E} u_{\mathcal{I}}\right) \mathrm{d} s+\int_{\partial E}\left(\boldsymbol{\beta} \cdot \boldsymbol{n}^{E}\right) e_{\mathcal{I}} e_{h} \mathrm{~d} s \\
& \lesssim \beta_{E}\left(\left\|e_{\mathcal{I}}\right\|_{L^{2}(\partial E)}+\left\|u-\Pi_{k}^{0, E} u_{\mathcal{I}}\right\|_{L^{2}(\partial E)}\right)\left\|\left(I-\Pi_{k}^{0, E}\right) e_{h}\right\|_{L^{2}(\partial E)}+\int_{\partial E}\left(\boldsymbol{\beta} \cdot \boldsymbol{n}^{E}\right) e_{\mathcal{I}} e_{h} \mathrm{~d} s \\
& \lesssim \beta_{E} h_{E}^{-1}\left(\left\|e_{\mathcal{I}}\right\|_{1, E}+\left\|u-\Pi_{k}^{0, E} u_{\mathcal{I}}\right\|_{1, E}\right)\left\|\left(I-\Pi_{k}^{0, E}\right) e_{h}\right\|_{0, E}+\int_{\partial E}\left(\boldsymbol{\beta} \cdot \boldsymbol{n}^{E}\right) e_{\mathcal{I}} e_{h} \mathrm{~d} s \\
& \lesssim \beta_{E}\left(\left\|e_{\mathcal{I}}\right\|_{1, E}+\left\|u-\Pi_{k}^{0, E} u\right\|_{1, E}\right)\left\|\nabla\left(I-\Pi_{k}^{\nabla, E}\right) e_{h}\right\|_{0, E}+\int_{\partial E}\left(\boldsymbol{\beta} \cdot \boldsymbol{n}^{E}\right) e_{\mathcal{I}} e_{h} \mathrm{~d} s \\
& \lesssim \sigma_{E} h_{E}^{s+1}|u|_{s+1, E}\left\|e_{h}\right\|_{\operatorname{supg}, E}+\int_{\partial E}\left(\boldsymbol{\beta} \cdot \boldsymbol{n}^{E}\right) e_{\mathcal{I}} e_{h} \mathrm{~d} s .
\end{aligned}
$$


The thesis now follows gathering (5.28), (5.29) and (5.30) in (5.27).

Remark 5.3. The main difficulty in proving Lemmas 5.6 and 5.7 lays in handling a variable coefficient $\boldsymbol{\beta}$ in the presence of projection operators, without paying a price for small values of $\varepsilon$. For form (4.5), we are able to obtain a "damped" dependence on $\varepsilon$ : in estimate (5.18) the term $\varepsilon^{-1 / 2}|\boldsymbol{\beta}|_{\left[W_{\infty}^{1}(E)\right]^{2}} h_{E}^{s+2}|u|_{s+1, E}$ blows up as $\varepsilon \rightarrow 0$, but at the same time it is of higher order with respect to $h_{E}$. Instead, for the new form (4.6) we are able to obtain full independence from $\varepsilon$.

We are now ready to prove the convergence results for the proposed VEM SUPG scheme. The error estimates in Lemmas 5.1-5.7 are explicit in the parameters of interest: the mesh size $h$, the diffusive coefficient $\varepsilon$, the advective field $\beta$ and the SUPG parameter $\tau_{E}$. In order to simplify the final estimate and to make clearer the implications of the convergence results, in the following propositions we include the Sobolev regularity terms for $u, f$ and the normalized norms $\frac{\|\boldsymbol{\beta}\|_{\left[W_{p}^{m}(E)\right]^{2}}}{\beta_{E}}$ in the constant.

Proposition 5.3. Under the assumptions (A.1) and (A.2), let $u \in V$ be the solution of equation (2.1) and $u_{h} \in V_{h}\left(\Omega_{h}\right)$ be the solution of equation (4.14) obtained with the bilinear form $b_{o, h}^{E}(\cdot, \cdot)$ in (4.5). Then it holds that

$$
\begin{aligned}
\left\|u-u_{h}\right\|_{\text {supg }}^{2} \lesssim \sum_{E \in \Omega_{h}} \Theta_{o}^{E}\left(h_{E}^{2 s}\left(\varepsilon+\tau_{E} \beta_{E}^{2}+\tau_{E}\right)+\lambda_{E}^{2} h_{E}^{2(s+2)}+\lambda_{E}^{2} \beta_{E}^{2} h_{E}^{2(s+1)}+\right. & \\
& \left.+\tau_{E} \varepsilon^{2} h_{E}^{2(s-1)}+\beta_{E}^{2} \frac{h_{E}^{2(s+2)}}{\varepsilon}+\beta_{E}^{2} \frac{h^{2(2 s+1)}}{\varepsilon}\right),
\end{aligned}
$$

where the constant $\Theta_{o}^{E}$ depends on $\|u\|_{s+1, E},\|f\|_{s+1, E}, \frac{\|\beta\|_{\left[W_{\infty}^{s+1}(E)\right]^{2}}}{\beta_{E}}$.

Proof. The proof is a direct consequence of Proposition 5.2, Lemmas 5.1, 5.3, 5.4, 5.5, and 5.6, making use of $\sigma_{E}=\beta_{E} \lambda_{E}$ and estimating the last two terms in (5.18) as follows.

The penultimate term is bounded using the Poincaré inequality on the domain $\Omega$

$$
\begin{aligned}
& \sum_{E \in \Omega_{h}}|\boldsymbol{\beta}|_{\left[W_{\infty}^{s+1}(E)\right]^{2}} h_{E}^{2 s+1}|u|_{s+1, E}\left\|e_{h}\right\|_{0, E} \\
& \leq\left(\sum_{E \in \Omega_{h}} \beta_{E}^{2}\left|\boldsymbol{\beta} / \beta_{E}\right|_{\left[W_{\infty}^{s+1}(E)\right]^{2}}^{2} h_{E}^{2(2 s+1)}|u|_{s+1, E}^{2}\right)^{1 / 2}\left(\sum_{E \in \Omega_{h}}\left\|e_{h}\right\|_{0, E}^{2}\right)^{1 / 2} \\
& \lesssim\left(\sum_{E \in \Omega_{h}} \beta_{E}^{2} h_{E}^{2(2 s+1)}|u|_{s+1, E}^{2}\right)^{1 / 2}\left\|e_{h}\right\|_{0, \Omega} \\
& \lesssim\left(\sum_{E \in \Omega_{h}} \beta_{E}^{2} h_{E}^{2(2 s+1)}|u|_{s+1, E}^{2}\right)^{1 / 2}\left\|\nabla e_{h}\right\|_{0, \Omega} \\
& \lesssim\left(\sum_{E \in \Omega_{h}} \beta_{E}^{2} \frac{h_{E}^{2(2 s+1)}}{\varepsilon}|u|_{s+1, E}^{2}\right)^{1 / 2}\left\|e_{h}\right\|_{\text {supg }} .
\end{aligned}
$$

For the last term, noticing that $e_{\mathcal{I}}, e_{h} \in V$, it holds that

$$
\sum_{E \in \Omega_{h}} \int_{\partial E}\left(\boldsymbol{\beta} \cdot \boldsymbol{n}^{E}\right) e_{\mathcal{I}} e_{h} \mathrm{~d} s=0
$$


Proposition 5.4. Under the assumptions (A.1) and (A.2), let $u \in V$ be the solution of equation (2.1) and $u_{h} \in V_{h}\left(\Omega_{h}\right)$ be the solution of equation (4.14) obtained with the bilinear form $b_{\partial, h}^{E}(\cdot, \cdot)$ in $(4.6)$. Then it holds that

$$
\left\|u-u_{h}\right\|_{\text {supg }}^{2} \lesssim \sum_{E \in \Omega_{h}} \Theta_{\partial}^{E}\left(h_{E}^{2 s}\left(\varepsilon+\tau_{E} \beta_{E}^{2}+\tau_{E}\right)+\lambda_{E}^{2} h_{E}^{2(s+2)}+\beta_{E}^{2} \lambda_{E}^{2} h_{E}^{2(s+1)}+\tau_{E} \varepsilon^{2} h_{E}^{2(s-1)}\right)
$$

where the constant $\Theta_{\partial}^{E}$ depends on $\|u\|_{s+1, E},\|f\|_{s+1, E}, \frac{\|\beta\|_{\left[W_{\infty}^{s+1}(E)\right]^{2}}}{\beta_{E}}$.

Proof. The proof follows from Proposition 5.2, Lemmas 5.1, 5.3, 5.4, 5.5, 5.7 and equation (5.31), also recalling that $\sigma_{E}=\beta_{E}^{2} \lambda_{E}^{2}$.

It is well known that in order to obtain a stable and optimal convergent method both in the convective and in the diffusion dominated regime the SUPG parameter $\tau_{E}$ has to be chosen in accordance with

$$
\tau_{E} \simeq \min \left\{\frac{h_{E}}{\beta_{E}}, \frac{h_{E}^{2}}{\varepsilon}\right\} .
$$

Let us analyse the asymptotic order of convergence for the two versions of VEM scheme in both regimes (where we recall $\beta_{E} \lesssim 1$ for all $E \in \Omega_{h}$ due to the scaling choice (A.0).

$$
\begin{aligned}
& \text { - convection dominated regime } \varepsilon \ll h_{E} \beta_{E}: \tau_{E}=\beta_{E}^{-1} h_{E}, \lambda_{E}^{2}=\beta_{E}^{-1} h_{E}^{-1} \text {, } \\
& \text { - form } b_{o, h}^{E}(\cdot, \cdot) \\
& \left\|u-u_{h}\right\|_{\text {supg }}^{2} \lesssim \sum_{E \in \Omega_{h}}\left(h_{E}^{2 s+1}\left(\beta_{E}+\beta_{E}^{-1}\right)+\beta_{E}^{-1} h_{E}^{2 s+3}+\varepsilon h_{E}^{2 s}+\beta_{E}^{-1} \varepsilon^{2} h_{E}^{2 s-1}\right. \\
& \left.+\beta_{E}^{2} \varepsilon^{-1} h_{E}^{2(s+2)}+\beta_{E}^{2} \varepsilon^{-1} h_{E}^{2(2 s+1)}\right)=O\left(h^{2 s+1}\left(1+\varepsilon^{-1} h^{3}\right)\right) ; \\
& -\operatorname{form} b_{\partial, h}^{E}(\cdot, \cdot) \\
& \left\|u-u_{h}\right\|_{\text {supg }}^{2} \lesssim \sum_{E \in \Omega_{h}}\left(h_{E}^{2 s+1}\left(\beta_{E}+\beta_{E}^{-1}\right)+\beta_{E}^{-1} h_{E}^{2 s+3}+\varepsilon h_{E}^{2 s}+\beta_{E}^{-1} \varepsilon^{2} h_{E}^{2 s-1}\right)=O\left(h^{2 s+1}\right) ; \\
& \text { - diffusion dominated regime } \beta_{E} h_{E} \ll \varepsilon: \tau_{E}=h_{E}^{2} \varepsilon^{-1}, \lambda_{E}^{2}=\varepsilon^{-1} \text {, } \\
& \text { - form } b_{o, h}^{E}(\cdot, \cdot) \\
& \left\|u-u_{h}\right\|_{\text {supg }}^{2} \lesssim \sum_{E \in \Omega_{h}}\left(\varepsilon h_{E}^{2 s}+\beta_{E}^{2} \varepsilon^{-1} h_{E}^{2(s+1)}+\varepsilon^{-1} h_{E}^{2(s+2)}\right)=O\left(\varepsilon h^{2 s}\right) \\
& \text { - form } b_{\partial, h}^{E}(\cdot, \cdot) \\
& \left\|u-u_{h}\right\|_{\text {supg }}^{2} \lesssim \sum_{E \in \Omega_{h}}\left(\varepsilon h_{E}^{2 s}+\beta_{E}^{2} \varepsilon^{-1} h_{E}^{2(s+1)}+\varepsilon^{-1} h_{E}^{2(s+2)}\right)=O\left(\varepsilon h^{2 s}\right) .
\end{aligned}
$$

We conclude that in the diffusion dominated regime both schemes yield the optimal rate of convergence. In the convection dominated regime only the scheme derived from the bilinear forms $b_{\partial, h}^{E}(\cdot, \cdot)$ has the optimal rate of convergence. For the scheme derived from $b_{o, h}^{E}(\cdot, \cdot)$ the error is polluted by $\varepsilon^{-1}$. Nevertheless, we stress that such a factor appears in front of the "higher" order term $h^{3}$, therefore the influence of the diffusion coefficient is strongly reduced.

\section{NUMERICAL EXPERIMENTS}

In this section we numerically validate the proposed methods by means of the following model problem. 

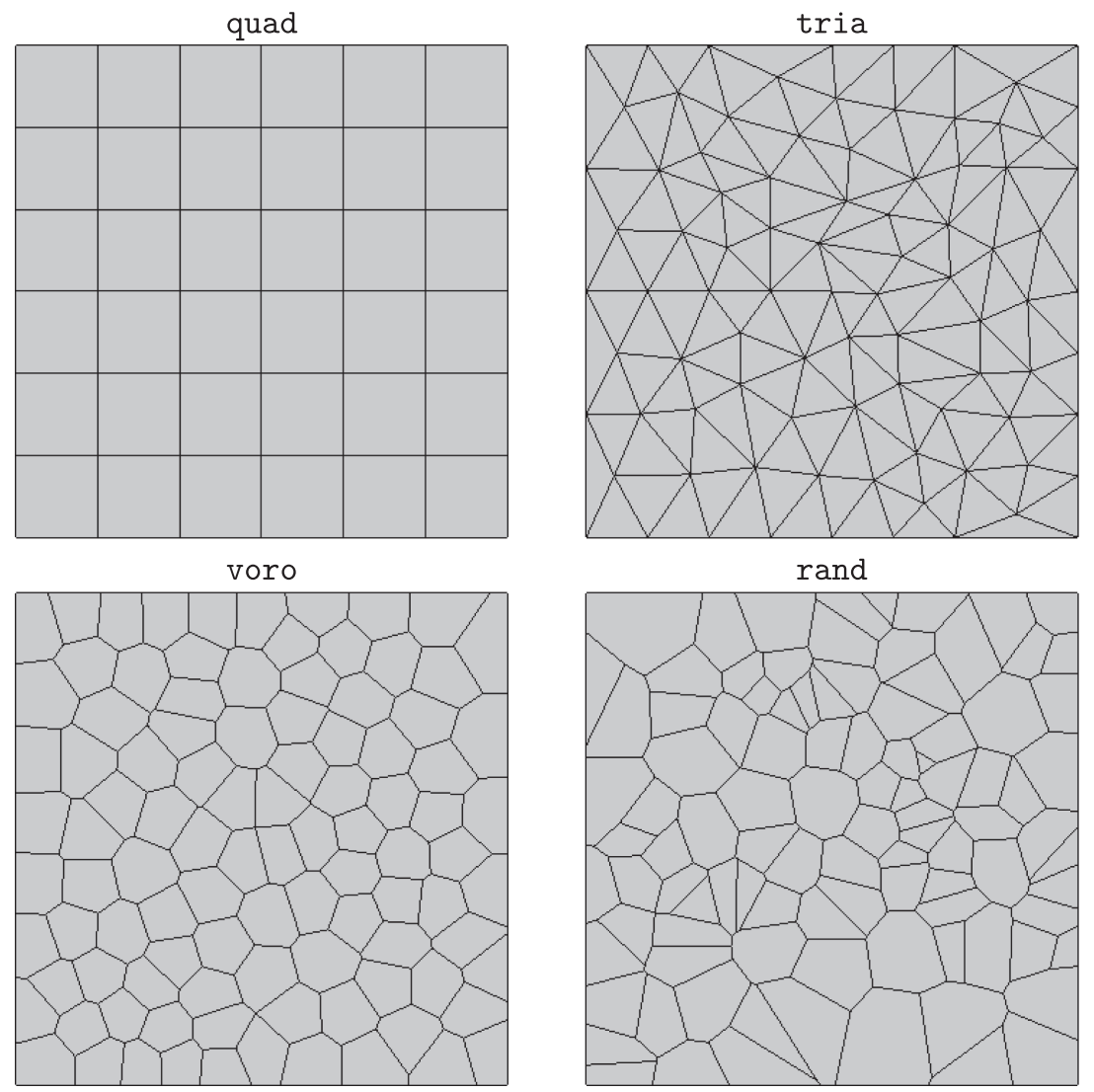

Figure 1. Example of meshes used for the present test case.

\section{Model problem}

We consider a family of problems in the unit square $\Omega=(0,1)^{2}$, one per each choice of the parameter $\varepsilon$. We select the advection term as

$$
\boldsymbol{\beta}(x, y):=\left[\begin{array}{r}
-2 \pi \sin (\pi(x+2 y)) \\
\pi \sin (\pi(x+2 y))
\end{array}\right] .
$$

We choose the boundary conditions and the source term (which turns out to depend on $\varepsilon$ ) in such a way that the analytical solution is always the function

$$
u(x, y):=\sin (\pi x) \sin (\pi y)
$$

Guided by the definition of the $\|\cdot\|_{\text {supg }}$ norm $(c f .(5.1)$ and (5.2)), by the error estimates of Propositions 5.35.4, and noticing that the discrete solution $u_{h} \in V_{h}\left(\Omega_{h}\right)$ is not explicitly point-wise available, the following error quantities will be considered.

- $H^{1}$-seminorm error

$$
e_{H^{1}}:=\sqrt{\sum_{E \in \mathcal{T}_{h}}\left\|\nabla\left(u-\Pi_{k}^{\nabla} u_{h}\right)\right\|_{0, E}^{2}} ;
$$




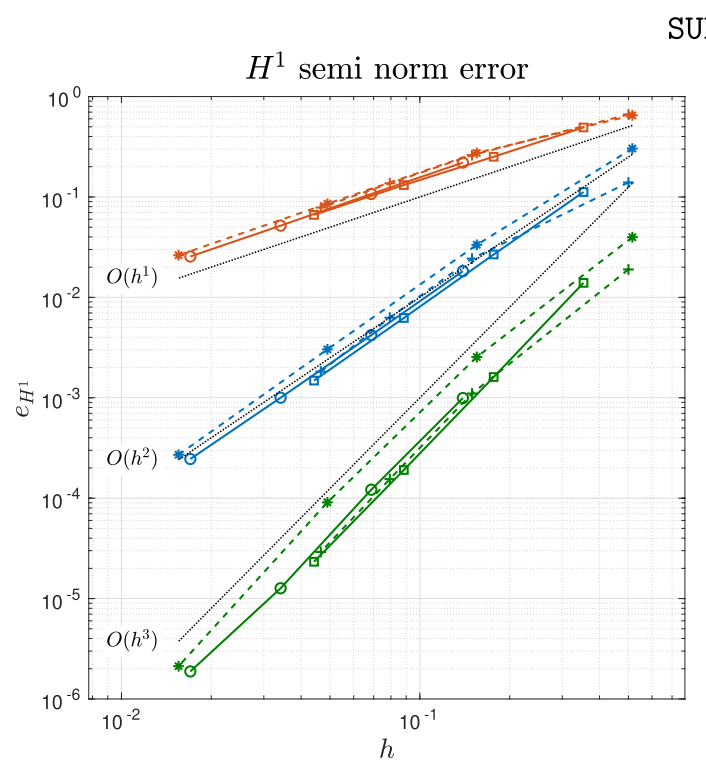

SUPG

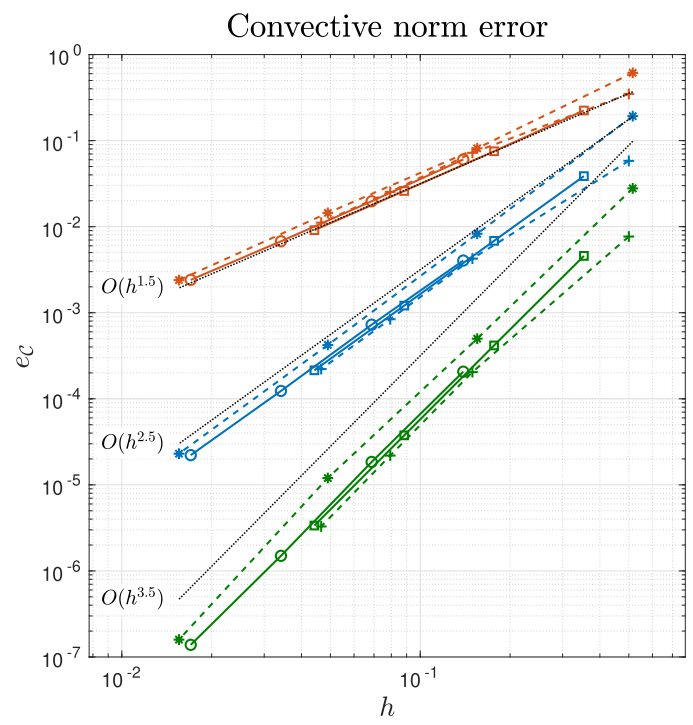

NONE

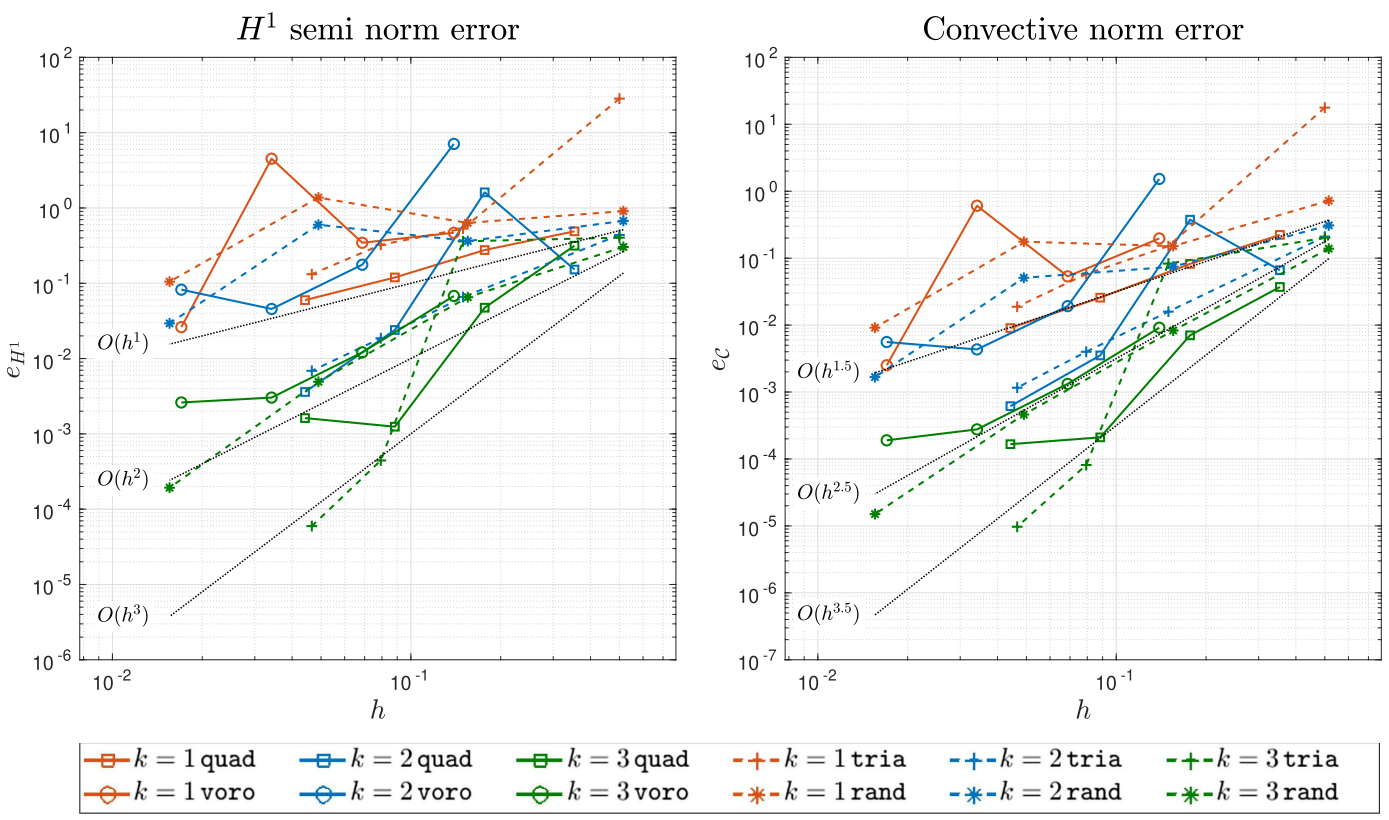

Figure 2. Effect of the SUPG term on the convergence histories: the case $\varepsilon=10^{-3}$.

- convective norm error

$$
e_{\mathcal{C}}:=\sqrt{\sum_{E \in \mathcal{T}_{h}}\left(\varepsilon\left\|\nabla\left(u-\Pi_{k}^{\nabla} u_{h}\right)\right\|_{0, E}^{2}+\tau_{E}\left\|\boldsymbol{\beta} \cdot \nabla\left(u-\Pi_{k}^{\nabla} u_{h}\right)\right\|_{0, E}^{2}\right)} .
$$

As far as the mesh types are concerned, we take the following:

- quad: a mesh composed by structured quadrilaterals; 


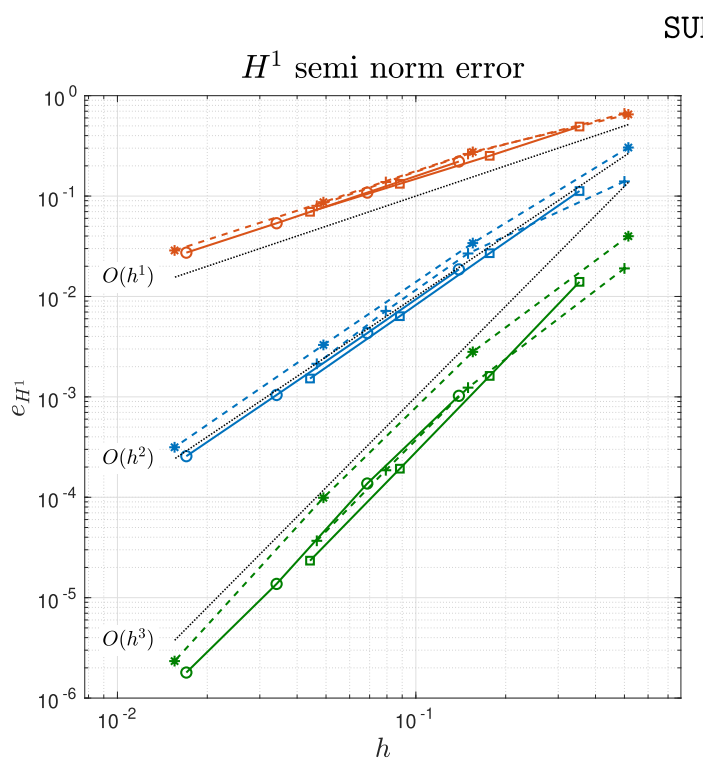

SUPG

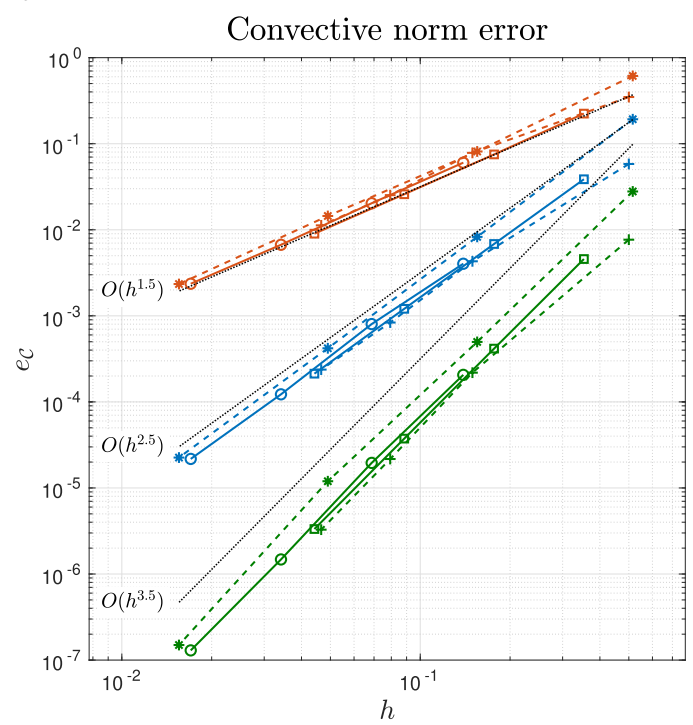

NONE

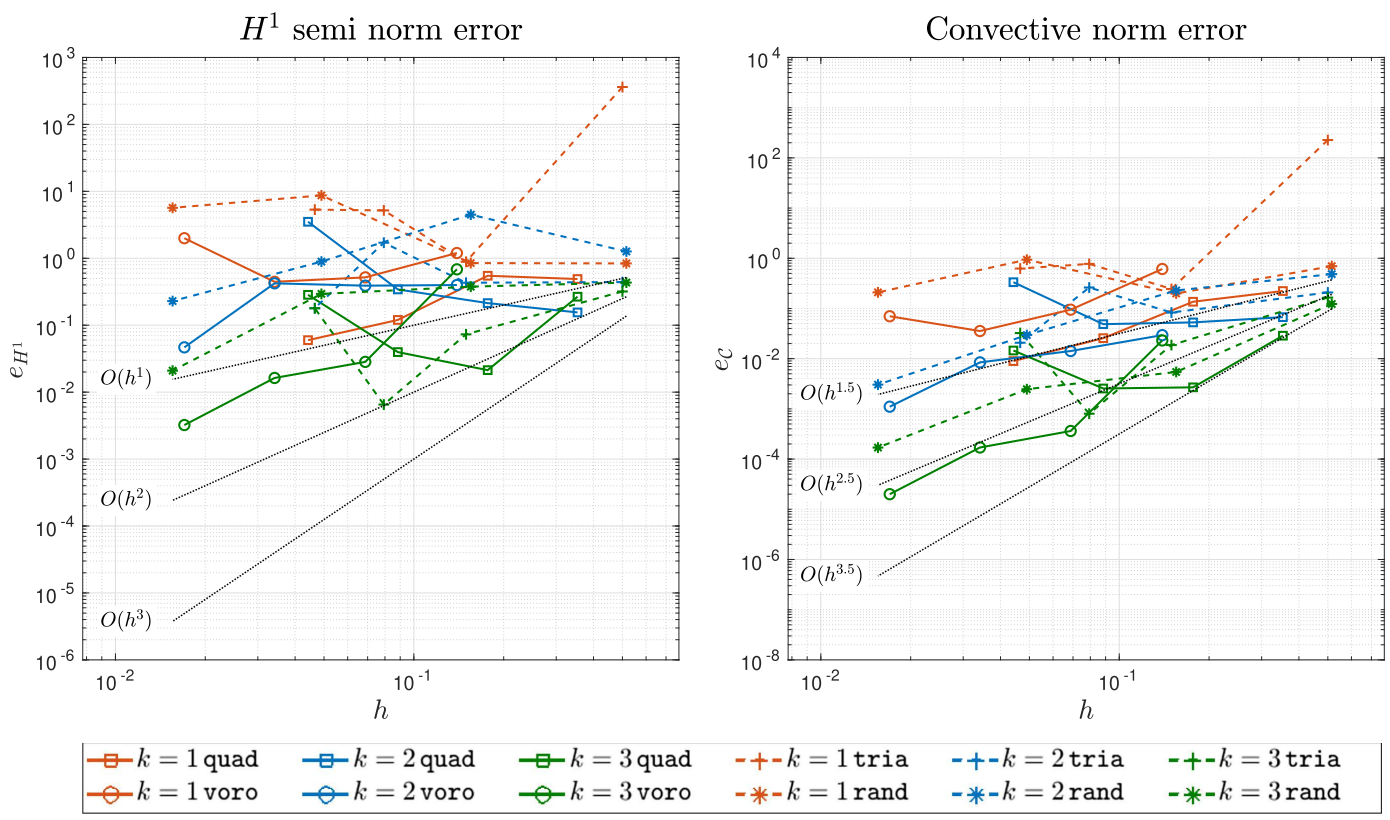

FiguRE 3. Effect of the SUPG term on the convergence histories: the case $\varepsilon=10^{-6}$.

- tria: a Delaunay triangulation of the unit square;

- voro: a centroidal Voronoi tessellation of the unit square where the cell shapes are optimized via a Lloyd algorithm

- rand: a Voronoi tessellation of the unit square where the cell shapes are not optimized.

In Figure 1 we show an example of such meshes, and we also remark that the former two types can be used in connection with a standard finite element procedure, contrary to the latter two. 


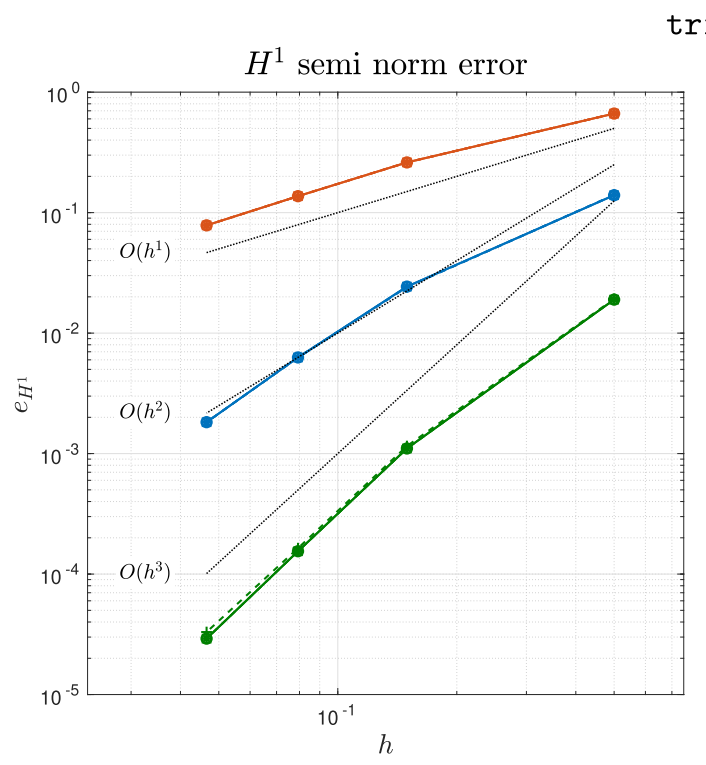

tria

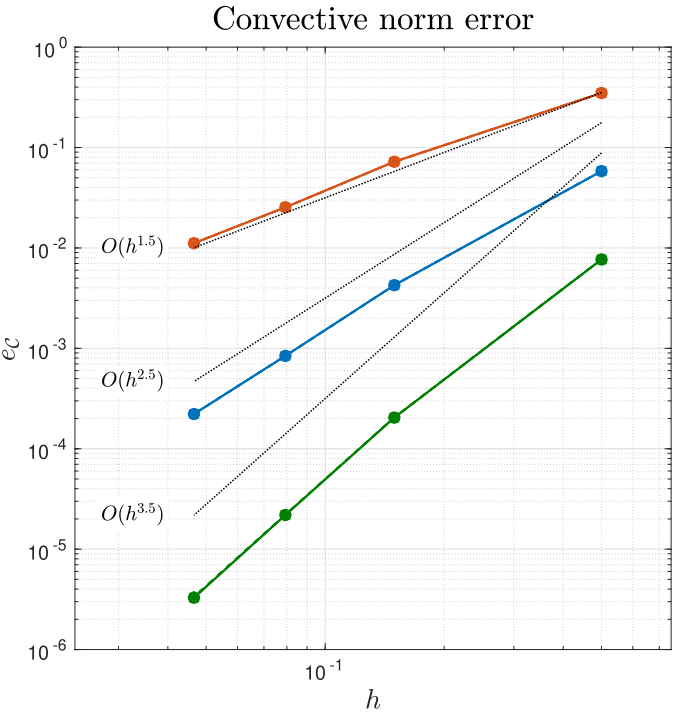

rand
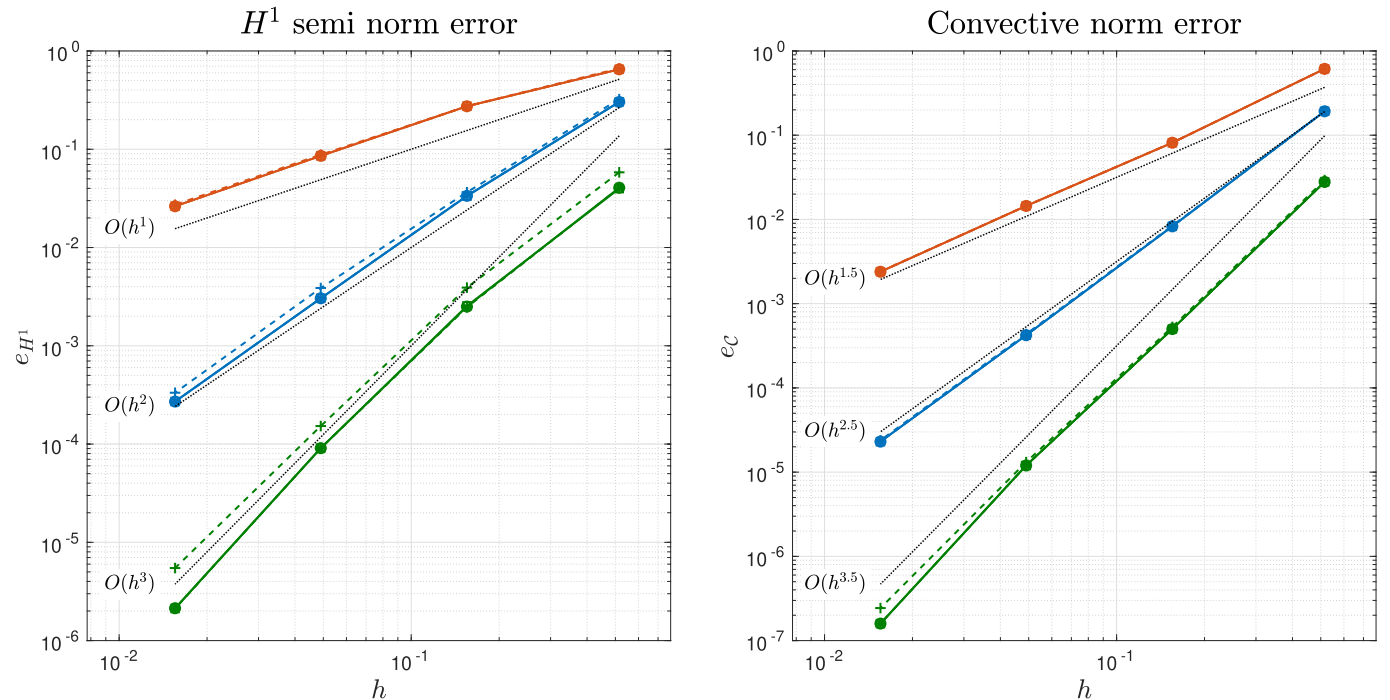

$\begin{array}{|llll|}-\square-k=1 \text { orig } & -+-k=1 \text { origSkew } & \ominus-k=1 \text { boun } & -*-k=1 \text { bounSkew } \\ \square-k=2 \text { orig } & -+-k=2 \text { origSkew } & -k=2 \text { boun } & -*-k=2 \text { bounSkew } \\ \square-k=3 \text { orig } & -+-k=3 \text { origSkew } & \ominus k=3 \text { boun } & -*-k=3 \text { bounSkew }\end{array}$

Figure 4 . Convergence lines for the case $\varepsilon=10^{-3}$, mesh types tria and rand.

Moreover, since we are interested in a robustness analysis with respect to the diffusion parameter, for each mesh sequence we take

$$
\varepsilon=10^{-3} \text { and } 10^{-6} \text {. }
$$

We recall that, for the stabilized scheme here proposed, in the presence of a regular solution the expected convergence rate in the convective norm is, in the convection dominated regime and for practical mesh ranges, 


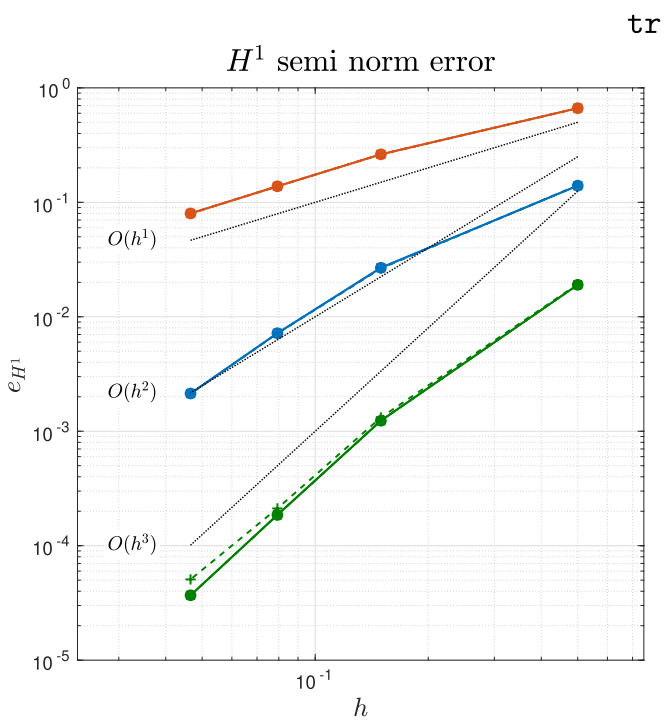

tria
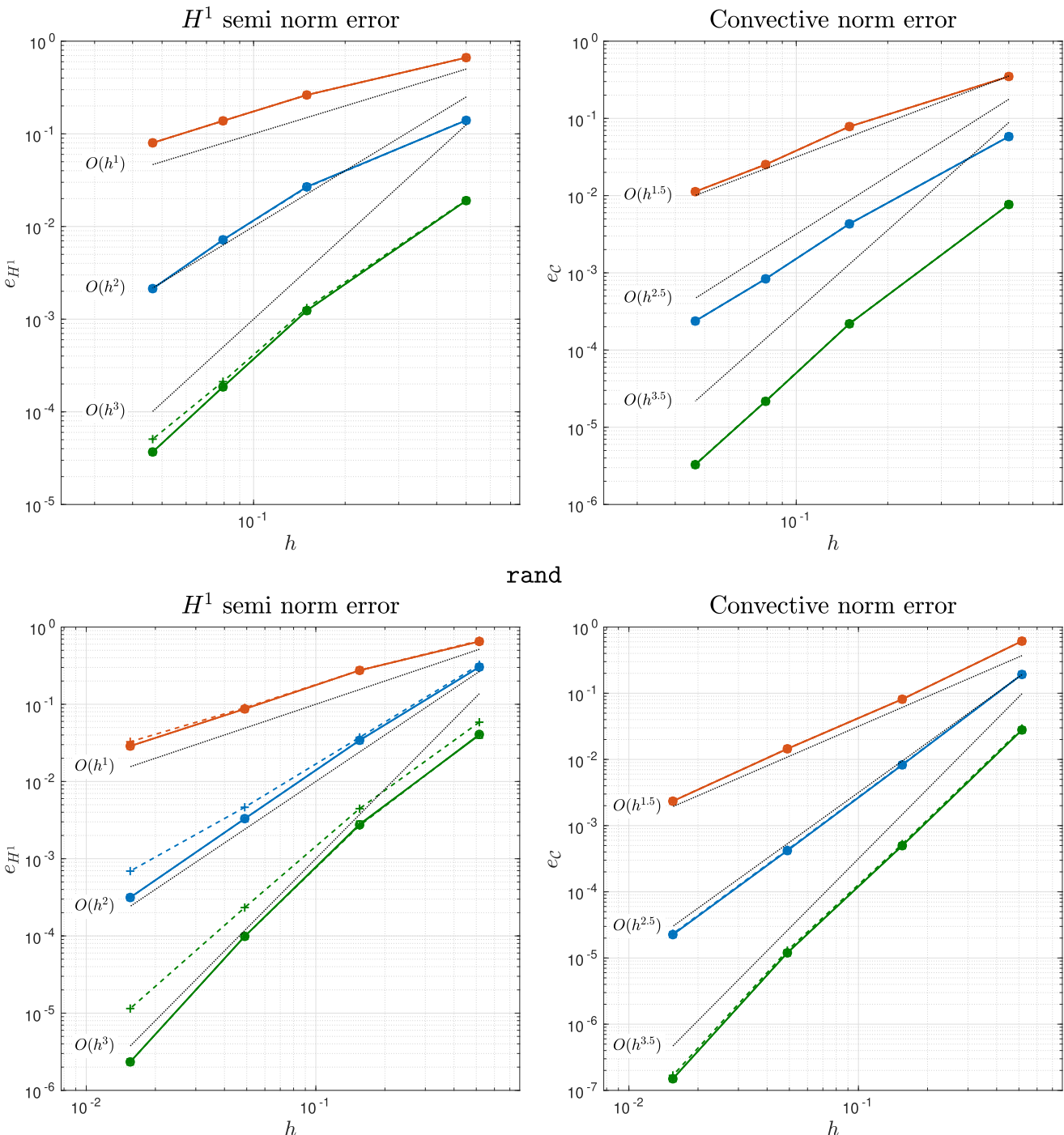

rand

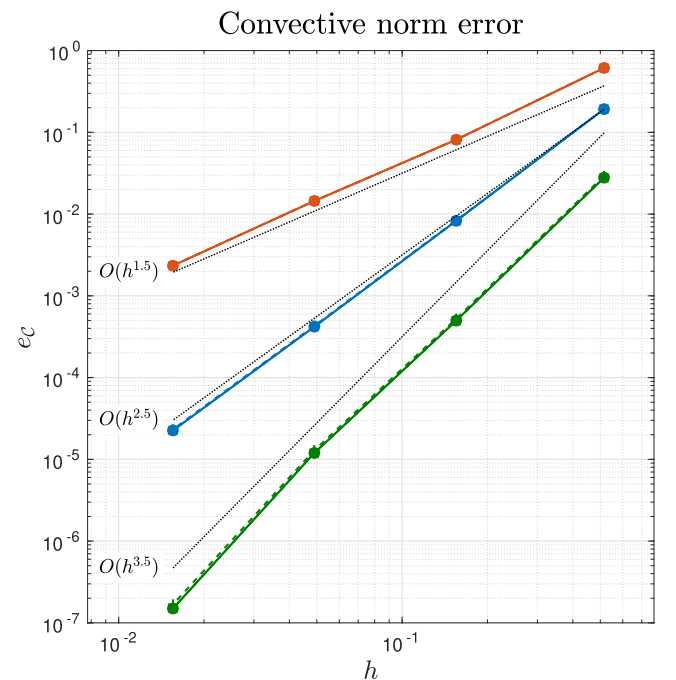

$\begin{array}{llll}\square-k=1 \text { orig } & -+-k=1 \text { origSkew } & -k=1 \text { boun } & - \text { * } k=1 \text { bounSkew } \\ \square-k=2 \text { orig } & -+-k=2 \text { origSkew } & -k=2 \text { boun } & - \text { *- } k=2 \text { bounSkew } \\ \square-k=3 \text { orig } & -+-k=3 \text { origSkew } & -k=3 \text { boun } & - \text { * } k=3 \text { bounSkew }\end{array}$

FiguRE 5. Convergence lines for the case $\varepsilon=10^{-6}$, mesh types tria and rand.

$O\left(h^{k+1 / 2}\right)$. This justifies the slope lines plotted in the relevant figures. For the $H^{1}$ norm, we instead plot standard $O\left(h^{k}\right)$ slopes, which is the best one can expect from the interpolation estimates.

\section{Effect of the SUPG stabilization}

Before assessing the convergence properties of the proposed methods, we check the effect of inserting the SUPG term in the variational formulation of the problem. Here we focus on the first choice for the convective bi-linear form, without the algebraical skew-symmetrization; namely we here use form (4.5). 
TABLE 1. Diffference between the stiffness matrices considering $b_{o, h}^{E}(\cdot, \cdot)$ or $b_{\partial, h}^{E}(\cdot, \cdot)$.

\begin{tabular}{l|c|c}
\hline \hline$k$ & \multicolumn{2}{|c}{ voro } \\
\hline 1 & $1.1221 \mathrm{e}-15$ & $1.7536 \mathrm{e}-17$ \\
2 & $9.2636 \mathrm{e}-16$ & $2.3161 \mathrm{e}-17$ \\
\hline
\end{tabular}

In Figures 2 and 3 we show the convergence graphs for different approximation degrees $k$ and for each type of the meshes. The cases with and without the SUPG term are labelled as SUPG and NONE, respectively. As expected, dropping the SUPG term clearly deteriorates the quality of the discrete solutions for all approximation degree $k$, the degeneration being heavier for smaller $\varepsilon$. We do not report the results for "large" $\varepsilon$, but we have experienced that, of course, both the SUPG and the NONE approaches give similar outcomes, attaining the correct convergence rate.

Incidentally, we remark that Figures 2 and 3 also display the robustness of the present SUPG virtual element approach with respect to element shape and distortion. In fact, given an approximation degree $k$, the convergence histories are rather similar for all the mesh types, even though the rand meshes are less structured and contain edges that are very small with respect to the element diameter.

\section{Different discrete convective bi-linear forms}

We now present the numerical results for different variants of the convective term approximation. More precisely, we consider four types of (local) discrete convective terms: $b_{o, h}^{E}(\cdot, \cdot)$ and $b_{\partial, h}^{E}(\cdot, \cdot)$, and their skew-symmetric counterparts, see (4.5), (4.6) and (4.7). We refer to such approximations as orig and boun, respectively, while we label their skew-symmetric counterparts as origSkew and bounSkew (the ones covered by the theoretical analysis developed in Sect. 5).

To avoid instabilities in the convection dominated regime, in what follows we always take advantage of the SUPG stabilization.

In Figures 4 and 5 we collect the results with the diffusion coefficients $\varepsilon=10^{-3}$ and $\varepsilon=10^{-6}$, respectively. We here show the results only for the mesh types tria and rand since the results obtained with the other types are very similar. We notice that the convergence rate of both error norms $e_{\mathcal{C}}$ and $e_{H^{1}}$ are the expected ones, and they are robust in the parameter $\varepsilon$. When we consider the $H^{1}$-seminorm error $e_{H^{1}}$, we observe that the origSkew behaves slightly worst than the other three discrete forms, especially for the approximation degrees $k=2,3$, and independently of the values of $\varepsilon$.

We conclude the section by noticing that $b_{\partial, h}^{E}(\cdot, \cdot)$ and $b_{o, h}^{E}(\cdot, \cdot)$ coincide for a constant convection term $\boldsymbol{\beta}$ (the proof can be easily performed by a direct computation). To have a numerical evidence about this fact, we have considered a problem with constant vector field $\boldsymbol{\beta}$ and compared the stiffness matrices provided by $b_{o, h}^{E}(\cdot, \cdot)$ and $b_{\partial, h}^{E}(\cdot, \cdot)$, respectively. For every mesh and every approximation degree, we have found that they always differ up to machine precision. Here we show the data (norms of the difference between the stiffness matrices) only for the finest voro mesh and for $k=1,2$, see Table 1 .

Acknowledgements. The authors L. BdV, F.D. and G.V. were partially supported by the European Research Council through the H2020 Consolidator Grant (grant no. 681162) CAVE, "Challenges and Advancements in Virtual Elements". This support is gratefully acknowledged. The authors L. BdV and C.L. were partially supported by the italian PRIN 2017 grant "Virtual Element Methods: Analysis and Applications". This support is gratefully acknowledged.

\section{REFERENCES}

[1] R.A. Adams, Sobolev spaces, In Vol. 65 of Pure and Applied Mathematics. Academic Press, New York-London (1975). 
[2] B. Ahmad, A. Alsaedi, F. Brezzi, L.D. Marini and A. Russo, Equivalent projectors for virtual element methods. Comput. Math. Appl. 66 (2013) 376-391.

[3] P.F. Antonietti, S. Giani and P. Houston, Hp-Version composite discontinuous Galerkin methods for elliptic problems on complicated domains. SIAM J. Sci. Comput. 35 (2013) A1417-A1439.

[4] P.F. Antonietti, A. Cangiani, J. Collis, Z. Dong, E.H. Georgoulis, S. Giani and P. Houston, Review of discontinuous Galerkin finite element methods for partial differential equations on complicated domains. Lect. Notes Comput. Sci. Eng. 50 (2016) 699-725.

[5] B. Ayuso De Dios, K. Lipnikov and G. Manzini, The nonconforming virtual element method. ESAIM Math. Model. Numer. Anal. 50 (2016) 879-904.

[6] L. Beirão da Veiga and G. Vacca, Sharper error estimates for Virtual Elements and a bubble-enriched version (2020).

[7] L. Beirão da Veiga, F. Brezzi, A. Cangiani, G. Manzini, L.D. Marini and A. Russo, Basic principles of Virtual Element Methods. Math. Models Methods Appl. Sci. 23 (2013) 199-214.

[8] L. Beirão da Veiga, F. Brezzi, L.D. Marini and A. Russo, The Hitchhiker's Guide to the Virtual Element Method. Math. Models Methods Appl. Sci. 24 (2014) 1541-1573.

[9] L. Beirão da Veiga, F. Brezzi, L.D. Marini and A. Russo, Mixed virtual element methods for general second order elliptic problems on polygonal meshes. ESAIM Math. Model. Numer. Anal. 50 (2016) 727-747.

[10] L. Beirão da Veiga, F. Brezzi, L.D. Marini and A. Russo, Virtual Element Method for general second-order elliptic problems on polygonal meshes. Math. Models Methods Appl. Sci. 26 (2016) 729-750.

[11] L. Beirão da Veiga, F. Dassi and A. Russo, High-order virtual element method on polyhedral meshes. Comput. Math. Appl. 74 (2017) 1110-1122.

[12] L. Beirão da Veiga, C. Lovadina and A. Russo, Stability analysis for the virtual element method. Math. Models Methods Appl. Sci. 27 (2017) 2557-2594.

[13] L. Beirão da Veiga, A. Pichler and G. Vacca, A virtual element method for the miscible displacement of incompressible fluids in porous media. Comput. Methods Appl. Mech. Eng. 375 (2021) 113649.

[14] M.F. Benedetto, S. Berrone, A. Borio, S. Pieraccini and S. Scialò, A hybrid mortar virtual element method for discrete fracture network simulations. J. Comput. Phys. 306 (2016) 148-166.

[15] M.F. Benedetto, S. Berrone, A. Borio, S. Pieraccini and S. Scialò, Order preserving SUPG stabilization for the virtual element formulation of advection-diffusion problems. Comput. Methods Appl. Mech. Eng. 293 (2016) 18-40.

[16] M.F. Benedetto, S. Berrone and S. Scialò, A globally conforming method for solving flow in discrete fracture networks using the Virtual Element Method. Finite Elem. Anal. Des. 109 (2016) 23-36.

[17] S. Berrone, A. Borio and G. Manzini, SUPG stabilization for the nonconforming virtual element method for advection-diffusionreaction equations. Comput. Methods Appl. Mech. Eng. 340 (2018) 500-529.

[18] S.C. Brenner and L.R. Scott, The mathematical theory of finite element methods, 3rd edition. In Vol. 15 of Texts in Applied Mathematics, Springer, New York (2008).

[19] S.C. Brenner and L.Y. Sung, Virtual element methods on meshes with small edges or faces. Math. Models Methods Appl. Sci. 28 (2018) 1291-1336.

[20] F. Brezzi, R. Falk and L.D. Marini, Basic principles of mixed virtual element methods. ESAIM Math. Model. Numer. Anal. 48 (2014) 1227-1240.

[21] A. Cangiani, E.H. Georgoulis and P. Houston, Hp-Version discontinuous Galerkin methods on polygonal and polyhedral meshes. Math. Models Methods Appl. Sci. 24 (2014) 2009-2041.

[22] A. Cangiani, Z. Dong, E.H. Georgoulis and P. Houston, Hp-Version discontinuous Galerkin methods for advection-diffusionreaction problems on polytopic meshes. ESAIM Math. Model. Numer. Anal. 50 (2016) 699-725.

[23] A. Cangiani, E.H. Georgoulis, T. Pryer and O.J. Sutton, A posteriori error estimates for the virtual element method. Numer. Math. 137 (2017) 857-893.

[24] A. Cangiani, G. Manzini and O. Sutton, Conforming and nonconforming virtual element methods for elliptic problems. IMA J. Numer. Anal. 37 (2017) 1317-1354.

[25] L. Chen and J. Huang, Some error analysis on virtual element methods. Calcolo 55 (2018).

[26] J. Coulet, I. Faille, V. Girault, N. Guy and N. Nataf, A fully coupled scheme using virtual element method and finite volume for poroelasticity. Comput. Geosci. 24 (2020) 381-403.

[27] D.A. Di Pietro and A. Ern, Hybrid high-order methods for variable-diffusion problems on general meshes. C. R. Math. Acad. Sci. Paris 353 (2015) 31-34.

[28] D.A. Di Pietro, J. Droniou and A. Ern, A discontinuous-skeletal method for advection-diffusion-reaction on general meshes. SIAM J. Numer. Anal. 53 (2015) 2135-2157.

[29] L.P. Franca, S.L. Frey and T. J. R. Hughes, Stabilized finite element methods. I. Application to the advective-diffusive model. Comput. Methods Appl. Mech. Eng. 95 (1992) 253-276.

[30] A. Fumagalli and E. Keilegavlen, Dual virtual element method for discrete fractures networks. SIAM J. Sci. Comput. 40 (2018) B228-B258.

[31] A. Fumagalli and E. Keilegavlen, Dual virtual element methods for discrete fracture matrix models. Oil Gas Sci. Technol. $\mathbf{7 4}$ (2019) 41 . 
[32] T.J.R. Hughes and A. N. Brooks, A theoretical framework for Petrov-Galerkin methods with discontinuous weighting functions: Application to the streamline-upwind procedure. Finite Elem. Fluids (1982) 47-65.

[33] A. Quarteroni and A. Valli, Numerical approximation of partial differential equations. Springer Science \& Business Media, Vol. 23 (2008).

\section{Subscribe to Open (S20) A fair and sustainable open access model}

This journal is currently published in open access under a Subscribe-to-Open model (S2O). S2O is a transformative model that aims to move subscription journals to open access. Open access is the free, immediate, online availability of research articles combined with the rights to use these articles fully in the digital environment. We are thankful to our subscribers and sponsors for making it possible to publish this journal in open access, free of charge for authors.

\section{Please help to maintain this journal in open access!}

Check that your library subscribes to the journal, or make a personal donation to the S2O programme, by contacting subscribers@edpsciences.org

More information, including a list of sponsors and a financial transparency report, available at: https://www. edpsciences.org/en/maths-s2o-programme 